\title{
Binding of $\mathrm{As}^{3+}$ and $\mathrm{As}^{5+}$ to $\mathrm{Fe}$ (III) Oxyhydroxide Clusters, and Influence of Aluminum Substitution: a Molecular Perspective (Supporting Information)
}

\author{
Sharmistha De Dalui and Bidisa Das ${ }^{1, *}$ \\ ${ }^{1}$ Technical Research Center (TRC). \\ School of Applied and Interdisciplinary Sciences (SAIS). \\ Indian Association for the Cultivation of Science (IACS). \\ 2A \& 2B Raja S C Mullick Road. Jadavpur. \\ Kolkata 700032. West Bengal. India.
}

The Gibb's free energies of formation for selected small clusters using 6-31G** and Augcc-pVTZ basis-sets

The use of B3LYP/6-31G** methods for this study was validated by performing few additional studies involving extended Aug-cc-pVTZ basis-set for selected small clusters, and the results are included in the below. We find that though the structures of the clusters are minimally affected by the change in basis-set, the reaction free energies are somewhat different.

Table S1. Comparison of bond distances (in A) for $\left[\mathrm{Fe}_{2}(\mathrm{OH})_{2}\left(\mathrm{H}_{2} \mathrm{O}\right)_{8}\right]^{4+}$ calculated using two basis-sets

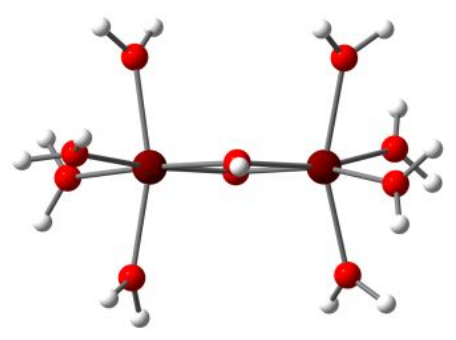

\begin{tabular}{|l|l|l|l|l|}
\hline Complex & $\begin{array}{l}\mathrm{Fe}-\mathrm{O} \\
\mathrm{H}(\mathrm{brd})\end{array}$ & Fe-OH ${ }_{2}$ ligands & $\mathrm{O}-\mathrm{H}$ (Bridge) & $\begin{array}{l}\text { Fe-Fe } \\
\text { (via }-\mathrm{OH})\end{array}$ \\
\hline $\begin{array}{l}{\left[\mathrm{Fe}_{\mathbf{2}}(\mathrm{OH})_{2}\left(\mathrm{H}_{\mathbf{2}} \mathrm{O}\right)_{8}\right]^{4+}} \\
\mathrm{B} 3 \mathrm{LYP} / 6-31 \mathrm{G}^{* *}\end{array}$ & $1.97-2.00$ & $2.07-2.09$ & 0.97 & 3.19 \\
\hline $\begin{array}{l}{\left[\mathrm{Fe}_{2}(\mathrm{OH})_{2}\left(\mathrm{H}_{\mathbf{2}} \mathrm{O}\right)_{8}\right]^{4+}} \\
\text { B3LYP/Aug-ccpVTZ }\end{array}$ & $1.98-2.03$ & $2.08-2.10$ & 0.97 & 3.23 \\
\hline
\end{tabular}

*Email:cambd@iacs.res.in 
Table S2. Comparison of Enthalpy and Gibb's free energy in both Gas phase and solution for $\left[\mathrm{Fe}_{2}(\mathrm{OH})_{2}\left(\mathrm{H}_{2} \mathrm{O}\right)_{8}\right]^{4+}$ calculated using two basis-sets.

\begin{tabular}{|l|l|l|l|l|}
\hline $\begin{array}{l}\text { lons } \\
\text { Interacting }\end{array}$ & Basis Set & Reactions & $\begin{array}{l}\Delta H, \Delta G \text { in } k c a l \\
\text { In Gas Phase }\end{array}$ & $\begin{array}{l}\Delta G_{s} \text { in } k c a l \\
\text { In water }\end{array}$ \\
\hline $\begin{array}{l}{\left[\mathrm{Fe}(\mathrm{OH})\left(\mathrm{H}_{2} \mathrm{O}\right)_{5}\right]^{2}} \\
+\end{array}$ & $\begin{array}{l}\text { UB3LYP/6- } \\
31 G^{* *}\end{array}$ & $2\left[\mathrm{Fe}(\mathrm{OH})\left(\mathrm{H}_{2} \mathrm{O}\right)_{5}\right]^{2+} \rightarrow\left[\mathrm{Fe}_{2}(\mathrm{OH})_{2}\left(\mathrm{H}_{2} \mathrm{O}\right)_{8}\right]^{4+}+2 \mathrm{H}_{2} \mathrm{O}$ & $\begin{array}{l}+263.56 \\
+259.63\end{array}$ & -8.42 \\
\hline $\begin{array}{l}{\left[\mathrm{Fe}(\mathrm{OH})\left(\mathrm{H}_{2} \mathrm{O}\right)_{5}\right]^{2}} \\
+\end{array}$ & $\begin{array}{l}\text { UB3LYP/Aug- } \\
c c-p V T Z\end{array}$ & $2\left[\mathrm{Fe}(\mathrm{OH})\left(\mathrm{H}_{2} \mathrm{O}\right)_{5}\right]^{2+} \rightarrow\left[\mathrm{Fe}_{2}(\mathrm{OH})_{2}\left(\mathrm{H}_{2} \mathrm{O}\right)_{8}\right]^{4+}+2 \mathrm{H}_{2} \mathrm{O}$ & +256.34 & -11.34 \\
& & +252.32 & \\
\hline
\end{tabular}

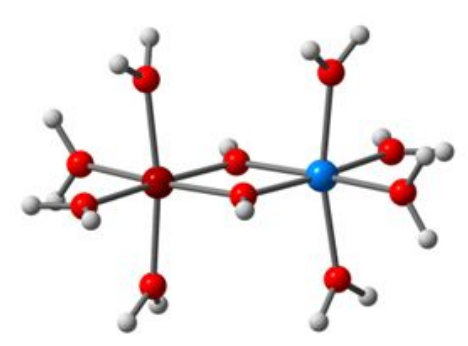

Table S3. Comparison of bond distances (in $A)$ for $\left[\mathrm{FeAl}(\mathrm{OH})_{2}\left(\mathrm{H}_{2} \mathrm{O}\right)_{8}\right]^{4+}$ calculated using two basis-sets.

\begin{tabular}{|c|c|c|c|c|c|c|}
\hline Complex & $\begin{array}{l}\mathrm{Fe}-\mathrm{O} \\
\mathrm{H} \text { (brd) }\end{array}$ & $\begin{array}{l}\mathrm{Al}-\mathrm{OH} \\
\text { (brd) }\end{array}$ & $\begin{array}{l}\mathrm{Fe}-\mathrm{OH}_{2} \\
\text { ligands }\end{array}$ & $\begin{array}{l}\mathrm{Al}-\mathrm{OH}_{2} \\
\text { ligands }\end{array}$ & $\begin{array}{l}\mathrm{O}-\mathrm{H} \\
\text { (Bridge) }\end{array}$ & $\begin{array}{l}\text { Fe-Al } \\
\text { (via }-\mathrm{OH} \text { ) }\end{array}$ \\
\hline $\begin{array}{l}{\left[\mathrm{FeAl}(\mathrm{OH})_{2}\left(\mathrm{H}_{2} \mathrm{O}\right)_{8}\right]^{4+}} \\
\mathrm{B} 3 \mathrm{LYP} / 6-31 \mathrm{G}^{* *}\end{array}$ & $\begin{array}{l}1.97 \\
2.00\end{array}$ & $\begin{array}{l}1.88 \\
1.91\end{array}$ & $\begin{array}{l}2.07,2.08 \\
2.09\end{array}$ & 1.96 & 0.97 & 3.09 \\
\hline $\begin{array}{l}{\left[\mathrm{FeAl}(\mathrm{OH})_{2}\left(\mathrm{H}_{2} \mathrm{O}\right)_{8}\right]^{4+}} \\
\text { B3LYP/Aug-ccpVTZ }\end{array}$ & $\begin{array}{l}1.98 \\
2.03\end{array}$ & $\begin{array}{l}1.87 \\
1.91\end{array}$ & $\begin{array}{l}2.08 \\
2.10\end{array}$ & 1.97 & 0.97 & 3.11 \\
\hline
\end{tabular}

Table S4. Comparison of Enthalpy and Gibb's free energy in both Gas phase and solution for $\left[\mathrm{FeAl}(\mathrm{OH})_{2}\left(\mathrm{H}_{2} \mathrm{O}\right)_{8}\right]^{4+}$ calculated using two basis-sets.

\begin{tabular}{|c|c|c|c|c|}
\hline $\begin{array}{c}\text { Ions } \\
\text { Interacting }\end{array}$ & Basis Set & Reactions & $\begin{array}{l}\Delta H, \Delta G \text { in kcal } \\
\text { In Gas Phase }\end{array}$ & $\begin{array}{c}\Delta G_{s} \text { in } k c a l \\
\text { In water }\end{array}$ \\
\hline $\begin{array}{l}{\left[\mathrm{Fe}(\mathrm{OH})\left(\mathrm{H}_{2} \mathrm{O}\right)_{5}\right]^{2}} \\
+ \\
{\left[\mathrm{Al}(\mathrm{OH})\left(\mathrm{H}_{2} \mathrm{O}\right)_{5}\right]^{2+}}\end{array}$ & $\begin{array}{l}\text { UB3LYP/6- } \\
31 G^{* *}\end{array}$ & $\begin{array}{l}{\left[\mathrm{Fe}(\mathrm{OH})\left(\mathrm{H}_{2} \mathrm{O}\right)_{5}\right]^{2+}+\left[\mathrm{Al}(\mathrm{OH})\left(\mathrm{H}_{2} \mathrm{O}\right)_{5}\right]^{2+}} \\
\rightarrow\left[\mathrm{FeAl}(\mathrm{OH})_{2}\left(\mathrm{H}_{2} \mathrm{O}\right)_{8}\right]^{4+}+2 \mathrm{H}_{2} \mathrm{O}\end{array}$ & $\begin{array}{l}\Delta H=+251.70 \\
\Delta G=+246.03\end{array}$ & -12.24 \\
\hline $\begin{array}{l}{\left[\mathrm{Fe}(\mathrm{OH})\left(\mathrm{H}_{2} \mathrm{O}\right)_{5}\right]^{2}} \\
+ \\
{\left[\mathrm{Al}(\mathrm{OH})\left(\mathrm{H}_{2} \mathrm{O}\right)_{5}\right]^{2+}}\end{array}$ & $\begin{array}{l}\text { UB3LYP/Aug- } \\
\text { cc-pVTZ }\end{array}$ & $\begin{array}{l}{\left[\mathrm{Fe}(\mathrm{OH})\left(\mathrm{H}_{2} \mathrm{O}\right)_{5}\right]^{2+}+\left[\mathrm{Al}(\mathrm{OH})\left(\mathrm{H}_{2} \mathrm{O}\right)_{5}\right]^{2+}} \\
\rightarrow\left[\mathrm{FeAl}(\mathrm{OH})_{2}\left(\mathrm{H}_{2} \mathrm{O}\right)_{8}\right]^{4+}+2 \mathrm{H}_{2} \mathrm{O}\end{array}$ & $\begin{array}{l}\Delta H=+244.55 \\
\Delta G=+239.55\end{array}$ & -16.27 \\
\hline
\end{tabular}




\section{Effects of incorporating few explicit water molecules in second hydration shell of the dimeric oxyhydroxide, $\left[\mathrm{Fe}_{2}(\mathrm{OH})_{2}\left(\mathrm{H}_{2} \mathrm{O}\right)_{8}\right]^{4+}$}

It is understood that implicit solvation strategies, overlook not only the effects of hydrogen-bonding in the solvent molecules, but also the effects of solute-solvent hydrogen-bonding is not correctly represented, which might influence the calculated energies. To understand the effect, we have done some model studies using few explicit water molecules in the second hydration shell of the solute Fe-oxyhydroxide clusters. In this study, we have included a few explicit water molecules in the second hydration shell, in addition to the first octahedrally bound ones, to calculate the energies for the formation of dimeric $\mathrm{Fe}_{2}$ oxyhydroxide cluster.

For the monomer, two explicit water molecules in the second hydration shell is considered, so $\left[\mathrm{Fe}(\mathrm{OH})\left(\mathrm{H}_{2} \mathrm{O}\right)_{7}\right]^{2+}$ is the reactant ion and for the dimeric product four explicit water molecules were considered, $\left[\mathrm{Fe}_{\mathbf{2}}(\mathrm{OH})_{\mathbf{2}}\left(\mathrm{H}_{\mathbf{2}} \mathrm{O}\right)_{12}\right]^{4+}$ is the product. It is seen that for the dimeric $\mathrm{Fe}_{2}$ oxyhydroxide and dimeric Fe-Al oxyhydroxide cluster, there is only modest changes (1-2 kcal) in reaction Gibb's free energies after considering explicit water molecules in the second hydration shell. We also compared the few important bond-distances in the cases with single and double hydration shells, and we found that Fe-Fe and Fe-Al distances remain practically unchanged. Fe-Fe and Fe-Al distances are $3.19 \AA$ and $3.10 \AA$ in case of $\left[\mathrm{Fe}_{\mathbf{2}}(\mathbf{O H})_{\mathbf{2}}\left(\mathrm{H}_{\mathbf{2}} \mathbf{O}\right)_{12}\right]^{\mathbf{4}^{+}}$ $/\left[\mathrm{FeAl}(\mathrm{OH})_{\mathbf{2}}\left(\mathbf{H}_{2} \mathrm{O}\right)_{12}\right]^{4+}$ (with hydration shell, see scheme below) and for $\left[\mathrm{Fe}_{\mathbf{2}}(\mathrm{OH})_{\mathbf{2}}\left(\mathrm{H}_{\mathbf{2}} \mathrm{O}\right)_{8}\right]^{4+} /\left[\mathrm{FeAl}(\mathrm{OH})_{2}\left(\mathrm{H}_{2} \mathrm{O}\right)_{8}\right]^{4+}$, without second hydration shell; Fe-Fe:3.19 $\AA$, Fe-Al: $3.08 \AA$. Again for $\left[\mathrm{Fe}_{\mathbf{2}}(\mathrm{OH})_{\mathbf{2}}\left(\mathrm{H}_{\mathbf{2}} \mathrm{O}\right)_{12}\right]^{4+} /\left[\mathrm{FeAl}(\mathrm{OH})_{\mathbf{2}}\left(\mathrm{H}_{\mathbf{2}} \mathrm{O}\right)_{12}\right]^{4+}$ (with hydration shell, see figure below) the Fe-O, Al-O distances are $1.99 \AA$ and $1.89 \AA$ and for $\left[\mathrm{Fe}_{2}(\mathrm{OH})_{2}\left(\mathrm{H}_{2} \mathrm{O}\right)_{8}\right]^{4+} /\left[\mathrm{FeAl}(\mathrm{OH})_{2}\left(\mathrm{H}_{2} \mathrm{O}\right)_{8}\right]^{4+}$, without second hydration shell, the corresponding distances are FeO:1.98 $\AA$ and Al-O:1.89 $\AA$. We calculated the HOMO-LUMO gaps for the two systems and we find that $8.42 \mathrm{eV}$ gap for $\left[\mathrm{Fe}_{\mathbf{2}}(\mathrm{OH})_{\mathbf{2}}\left(\mathrm{H}_{\mathbf{2}} \mathrm{O}\right)_{12}\right]^{4+}$ with second hydration shell while HOMO-LUMO gap for $\left[\mathrm{Fe}_{\mathbf{2}}(\mathrm{OH})_{\mathbf{2}}\left(\mathrm{H}_{\mathbf{2}} \mathrm{O}\right)_{\mathbf{8}}\right]^{4+}$ is 8.71 $\mathrm{eV}$ (without hydration shell). In case of Fe-Al dimer the HOMO-LUMO gaps with second hydration shell is 8.73 $\mathrm{eV}$ and without second hydration shell is $9.13 \mathrm{eV}$. Therefore, we find no significant structural differences in the results with four explicit water molecules in the second hydration shell included, $\left[\mathrm{Fe}_{\mathbf{2}}(\mathbf{O H})_{\mathbf{2}}\left(\mathrm{H}_{\mathbf{2}} \mathbf{O}\right)_{\mathbf{1 2}_{2}}\right]^{4+}$ compared to none, $\left[\mathrm{Fe}_{2}(\mathrm{OH})_{2}\left(\mathrm{H}_{2} \mathrm{O}\right)_{8}\right]^{4+}$. 
Scheme S1. Effect of considering explicit water molecules in the second hydration shell for the formation of the dimeric oxyhydroxides. We had shown the solid blue circle for the first hydration shell and dotted blue circle marking the second hydration shell.
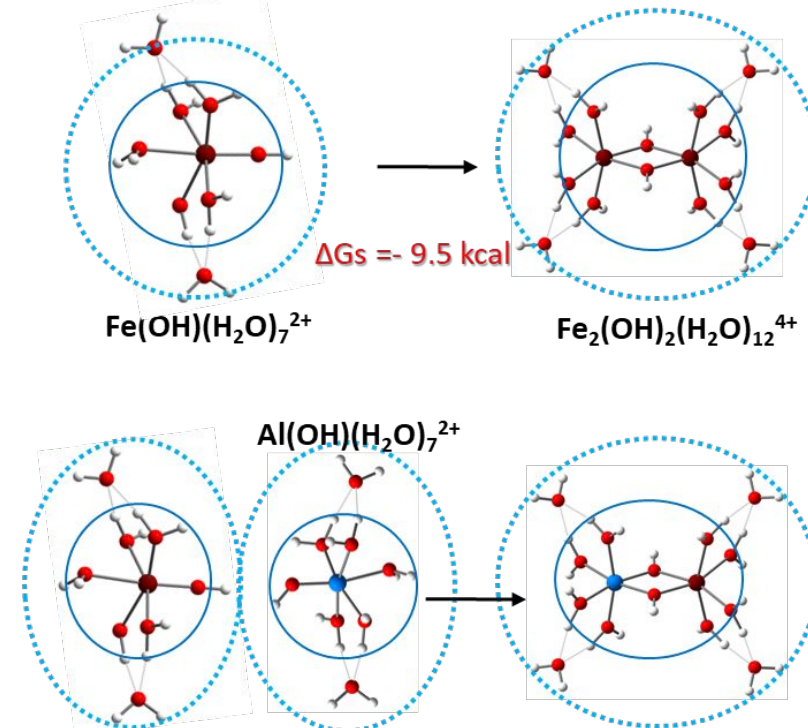

$\mathrm{Al}(\mathrm{OH})\left(\mathrm{H}_{2} \mathrm{O}\right)_{7}{ }^{2+}$

$\mathrm{Fe}(\mathrm{OH})\left(\mathrm{H}_{2} \mathrm{O}\right)_{7}^{2+}$

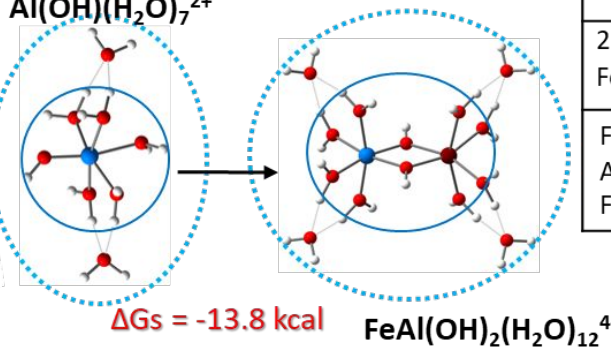

\begin{tabular}{|c|c|c|c|}
\hline Reactions & $\begin{array}{c}\Delta H \text { (gas) } \\
\text { (kcal) }\end{array}$ & $\begin{array}{c}\Delta \mathrm{G} \text { (gas) } \\
\text { (kcal) }\end{array}$ & $\begin{array}{r}\Delta \mathrm{G}_{\mathrm{s}} \\
\text { (kcal) }\end{array}$ \\
\hline \multicolumn{4}{|c|}{ Considering explicit water molecules in second hydration shell } \\
\hline $\begin{array}{l}2 \mathrm{Fe}(\mathrm{OH})\left(\mathrm{H}_{2} \mathrm{O}\right)_{7}^{2+} \rightarrow \\
\mathrm{Fe}_{2}(\mathrm{OH})_{2}\left(\mathrm{H}_{2} \mathrm{O}\right)_{12}{ }^{4+}+2 \mathrm{H}_{2} \mathrm{O}\end{array}$ & 203.55 & 199.9 & -9.5 \\
\hline $\begin{array}{l}\mathrm{Fe}(\mathrm{OH})\left(\mathrm{H}_{2} \mathrm{O}\right)_{7}{ }^{2+}+ \\
\mathrm{Al}(\mathrm{OH})\left(\mathrm{H}_{2} \mathrm{O}\right)_{7}^{2+} \rightarrow \\
\mathrm{FeAl}(\mathrm{OH})_{2}\left(\mathrm{H}_{2} \mathrm{O}\right)_{12}{ }^{4+}+2 \mathrm{H}_{2} \mathrm{O}\end{array}$ & 195.9 & 190.7 & -13.9 \\
\hline \multicolumn{4}{|c|}{ Considering only first hydration shell } \\
\hline $\begin{array}{l}2 \mathrm{Fe}(\mathrm{OH})\left(\mathrm{H}_{2} \mathrm{O}\right)_{5}{ }^{2+} \rightarrow \\
\mathrm{Fe}_{2}\left(\mathrm{H}_{2} \mathrm{O}\right)_{8}(\mathrm{OH})_{2}{ }^{4+}+2 \mathrm{H}_{2} \mathrm{O}\end{array}$ & 263.6 & 259.6 & -8.4 \\
\hline $\begin{array}{l}\mathrm{Fe}(\mathrm{OH})\left(\mathrm{H}_{2} \mathrm{O}\right)_{5}^{2+}+ \\
\mathrm{Al}(\mathrm{OH})\left(\mathrm{H}_{2} \mathrm{O}\right)_{5}{ }^{2+} \rightarrow \\
\mathrm{FeAl}(\mathrm{OH})_{2}\left(\mathrm{H}_{2} \mathrm{O}\right)_{8}^{4+}+2 \mathrm{H}_{2} \mathrm{O}\end{array}$ & 251.7 & 246.0 & -12.2 \\
\hline
\end{tabular}

\section{Gibb's free energies with $\mathrm{As}^{3+} / \mathrm{As}^{5+}$ ion binding to $\mathrm{Fe}(\mathrm{III})$ oxyhydroxide dimer}

The formation free energies of binding and important bond-distances of undoped pure Fe(III) oxyhydroxide dimer, $\left[\mathrm{Fe}_{2}(\mathrm{OH})_{2}\left(\mathrm{H}_{2} \mathrm{O}\right)_{8}\right]^{4+}$ in presence of $\mathbf{A s}(\mathrm{OH})_{3}, \mathbf{H}_{2} \mathrm{AsO}_{4}{ }^{-}, \mathrm{HAsO}_{4}^{-2}$ ions are listed below (B3LYP/6-31G**). Three inner sphere binding considered, (i) binuclear-bidentate (B-B) (ii) mononuclear-bidentate (M-B) (iii) mononuclear-monodentate (M-M).

Table S5. The reactions of $\mathrm{Fe}(\mathrm{III})$ oxyhydroxide dimeric clusters with $\mathrm{As}(\mathrm{OH})_{3}, \mathrm{HAsO}_{4}^{-}$and $\mathrm{HAsO}_{4}{ }^{2-i n}$ gas phase and in water are shown. Different complexation cases, the monodentate-mononuclear (M-M) bidentatebinuclear (B-B) and bidentate mononuclear (M-B) type are tabulated. Free energies for protonated and nonprotonated cases for $\mathrm{As}(\mathrm{OH})_{3}$ binding are also tabulated. In all cases, $\mathrm{Fe}^{3+}$ complexes are high-spin. The 
reaction free energies in aqueous medium are calculated form solvation free energies and corrected for standard states. ${ }^{17}$

\begin{tabular}{|c|c|c|c|c|}
\hline $\begin{array}{c}\text { Ions } \\
\text { Interacting }\end{array}$ & Reactions & $\begin{array}{l}\Delta G \text { in gas } \\
\text { phase } \\
\text { (in kcal) }\end{array}$ & $\begin{array}{l}\Delta \mathrm{G}_{\mathrm{s}} \text { in } \\
\text { water } \\
\text { (in kcal) }\end{array}$ & $\begin{array}{c}\text { Bond } \\
\text { Distances } \\
\text { (in Å) }\end{array}$ \\
\hline \multirow[t]{4}{*}{$\begin{array}{l}\mathrm{Fe}^{3+} \text { Dimer, } \\
\mathrm{As}(\mathrm{OH})_{3} \\
\text { Non- } \\
\text { protonated } \\
\text { products }\end{array}$} & $\begin{array}{l}{\left[\mathrm{Fe}_{2}(\mathrm{OH})_{2}\left(\mathrm{H}_{2} \mathrm{O}\right)_{8}\right]^{4+}+} \\
\mathrm{As}(\mathrm{OH})_{3} \rightarrow\left[\mathrm{Fe}_{2}(\mathrm{OH})_{2}\left(\mathrm{H}_{2} \mathrm{O}\right)_{6} \mathrm{AsO}_{2}(\mathrm{OH})\right]^{2+}+2 \mathrm{H}_{3} \mathrm{O}^{+}\end{array}$ & -294.15 & $\begin{array}{l}+5.90 \\
\text { (B-B) }\end{array}$ & $\begin{array}{l}\text { Fe-As: } \\
3.21-3.27 \\
\text { Fe-Fe: } \\
2.98 \\
\text { As-O: } \\
1.77-1.78 \\
\text { As-OH: } \\
1.86\end{array}$ \\
\hline & {$\left[\mathrm{Fe}_{2}(\mathrm{OH})_{2}\left(\mathrm{H}_{2} \mathrm{O}\right)_{8}\right]^{4+}+$} & -269.93 & $\begin{array}{l}+16.71 \\
\text { (M-B) }\end{array}$ & $\begin{array}{l}\text { Fe-As: } \\
2.65 \\
\text { Fe-Fe: } \\
3.12 \\
\text { As-O:1.80- } \\
1.81 \\
\text { As-OH: } \\
1.84\end{array}$ \\
\hline & {$\left[\mathrm{Fe}_{2}(\mathrm{OH})_{2}\left(\mathrm{H}_{2} \mathrm{O}\right)_{8}\right]^{4+}+$} & -285.41 & $\begin{array}{l}+13.60 \\
\text { (M-B) }\end{array}$ & $\begin{array}{l}\text { Fe-As: } \\
2.86 \\
\text { Fe-Fe: } \\
3.04 \\
\text { As-O: } \\
1.76 \\
\text { As-OH: } \\
1.79,1.87\end{array}$ \\
\hline & $\begin{array}{l}{\left[\mathrm{Fe}_{2}(\mathrm{OH})_{2}\left(\mathrm{H}_{2} \mathrm{O}\right)_{8}\right]^{4+}+} \\
\mathrm{As}(\mathrm{OH})_{3} \rightarrow\left[\mathrm{Fe}_{2}(\mathrm{OH})_{2}\left(\mathrm{H}_{2} \mathrm{O}\right)_{7} \mathrm{AsO}(\mathrm{OH})_{2}\right]^{3+} \\
+\mathrm{H}_{3} \mathrm{O}^{+}\end{array}$ & -219.12 & $\begin{array}{l}-4.54 \\
(\mathrm{M}-\mathrm{M})\end{array}$ & $\begin{array}{l}\text { Fe-As: } \\
3.42 \\
\text { Fe-Fe: } \\
3.12 \\
\text { As-O: } \\
1.76 \\
\text { As-OH: } \\
1.81,1.85\end{array}$ \\
\hline
\end{tabular}




\begin{tabular}{|c|c|c|c|c|}
\hline \multirow[t]{4}{*}{$\begin{array}{l}\mathrm{Fe}^{3+} \text { Dimer, } \\
\mathrm{As}(\mathrm{OH})_{3} \\
\text { Protonated } \\
\text { products }\end{array}$} & $\begin{array}{l}{\left[\mathrm{Fe}_{2}(\mathrm{OH})_{2}\left(\mathrm{H}_{2} \mathrm{O}\right)_{8}\right]^{4+}+} \\
\mathrm{As}(\mathrm{OH})_{3} \rightarrow\left[\mathrm{Fe}_{2}(\mathrm{OH})_{2}\left(\mathrm{H}_{2} \mathrm{O}\right)_{6} \mathrm{As}(\mathrm{OH})_{3}\right]^{4+}+2 \mathrm{H}_{2} \mathrm{O}\end{array}$ & -18.29 & $\begin{array}{l}-11.14 \\
\text { (B-B) }\end{array}$ & $\begin{array}{l}\text { Fe-As: } \\
3.64,3.65 \\
\text { Fe-Fe: } \\
3.11 \\
\text { As-OH: } \\
1.87,1.89 \\
1.76\end{array}$ \\
\hline & $\begin{array}{l}{\left[\mathrm{Fe}_{2}(\mathrm{OH})_{2}\left(\mathrm{H}_{2} \mathrm{O}\right)_{8}\right]^{4+}+} \\
\mathrm{As}(\mathrm{OH})_{3} \rightarrow\left[\mathrm{Fe}_{2}(\mathrm{OH})_{2}\left(\mathrm{H}_{2} \mathrm{O}\right)_{6} \mathrm{As}(\mathrm{OH})_{3}\right]^{4+}+2 \mathrm{H}_{2} \mathrm{O}\end{array}$ & -22.36 & $\begin{array}{l}-6.49 \\
(\mathrm{M}-\mathrm{B})\end{array}$ & $\begin{array}{l}\text { Fe-As: } \\
3.14 \\
\text { Fe-Fe: } \\
3.15 \\
\text { As-OH: } \\
1.89,1.76\end{array}$ \\
\hline & $\begin{array}{l}{\left[\mathrm{Fe}_{2}(\mathrm{OH})_{2}\left(\mathrm{H}_{2} \mathrm{O}\right)_{8}\right]^{4+}+} \\
\mathrm{As}(\mathrm{OH})_{3} \rightarrow\left[\mathrm{Fe}_{2}(\mathrm{OH})_{2}\left(\mathrm{H}_{2} \mathrm{O}\right)_{6} \mathrm{As}(\mathrm{OH})_{3}\right]^{4+}+2 \mathrm{H}_{2} \mathrm{O}\end{array}$ & -24.13 & $\begin{array}{l}-5.46 \\
\text { (M-B) }\end{array}$ & $\begin{array}{l}\text { Fe-As: } \\
3.16 \\
\text { Fe-Fe: } \\
3.17 \\
\text { As-OH: } \\
1.76,1.88 \\
1.89\end{array}$ \\
\hline & $\begin{array}{l}{\left[\mathrm{Fe}_{2}(\mathrm{OH})_{2}\left(\mathrm{H}_{2} \mathrm{O}\right)_{8}\right]^{4+}+} \\
\mathrm{As}(\mathrm{OH})_{3} \rightarrow\left[\mathrm{Fe}_{2}(\mathrm{OH})_{2}\left(\mathrm{H}_{2} \mathrm{O}\right)_{7} \mathrm{As}(\mathrm{OH})_{3}\right]^{4+}+\mathrm{H}_{2} \mathrm{O}\end{array}$ & -45.97 & $\begin{array}{l}-12.2 \\
(\mathrm{M}-\mathrm{M})\end{array}$ & $\begin{array}{l}\text { Fe-As: } \\
3.72 \\
\text { Fe-Fe: } \\
3.16 \\
\text { As-OH: } \\
1.78,1.80 \\
1.90\end{array}$ \\
\hline $\begin{array}{l}\mathrm{Fe}^{3+} \\
\mathrm{H}_{2} \mathrm{AsO}_{4}^{-}\end{array}$ & $\begin{array}{l}{\left[\mathrm{Fe}_{2}(\mathrm{OH})_{2}\left(\mathrm{H}_{2} \mathrm{O}\right)_{8}\right]^{4+}+\mathrm{H}_{2} \mathrm{AsO}_{4}^{-}} \\
\rightarrow\left[\mathrm{Fe}_{2}(\mathrm{OH})_{2}\left(\mathrm{H}_{2} \mathrm{O}\right)_{6} \mathrm{AsO}_{2}(\mathrm{OH})_{2}\right]^{3+}+2 \mathrm{H}_{2} \mathrm{O} \\
\end{array}$ & -388.22 & $\begin{array}{l}-44.34 \\
\text { (B-B) }\end{array}$ & $\begin{array}{l}\text { Fe-As: } \\
3.18 \\
\text { Fe-Fe: } \\
3.09 \\
\text { As-O: } \\
1.70 \\
\text { As-OH: } \\
1.70,1.72\end{array}$ \\
\hline
\end{tabular}




\begin{tabular}{|c|c|c|c|c|}
\hline & $\begin{array}{ll}\left.\mathrm{Fe}_{2}(\mathrm{OH})_{2}\left(\mathrm{H}_{2} \mathrm{O}\right)_{8}\right]^{1+}+\mathrm{H}_{2} \mathrm{AsO}_{4}^{-} \\
\rightarrow\left[\mathrm{Fe}_{2}(\mathrm{OH})_{2}\left(\mathrm{H}_{2} \mathrm{O}\right)_{6} \mathrm{AsO}_{2}(\mathrm{OH})_{2}\right]^{3+}+2 \mathrm{H}_{2} \mathrm{O}\end{array}$ & -366.14 & $\begin{array}{l}-33.16 \\
(\mathrm{M}-\mathrm{B})\end{array}$ & $\begin{array}{l}\text { Fe-As: } \\
2.64 \\
\text { Fe-Fe: } \\
3.11 \\
\text { As-O: } \\
1.72 \\
\text { As-OH: } \\
1.72\end{array}$ \\
\hline & {$\left[\mathrm{Fe}_{2}(\mathrm{OH})_{2}\left(\mathrm{H}_{2} \mathrm{O}\right)_{8}\right]^{4+}+\mathrm{H}_{2} \mathrm{AsO}_{4}^{-}$} & -372.91 & $\begin{array}{l}-32.58 \\
(M-B)\end{array}$ & $\begin{array}{l}\text { Fe-As: } \\
2.71 \\
\text { Fe-Fe: } \\
3.10 \\
\text { As-O: } \\
1.70,1.71 \\
\text { As-OH: } \\
1.71,1.72\end{array}$ \\
\hline & $\begin{array}{l}{\left[\mathrm{Fe}_{2}(\mathrm{OH})_{2}\left(\mathrm{H}_{2} \mathrm{O}\right)_{8}\right]^{4+}+\mathrm{H}_{2} \mathrm{AsO}_{4}^{-}} \\
\rightarrow\left[\mathrm{Fe}_{2}(\mathrm{OH})_{2}\left(\mathrm{H}_{2} \mathrm{O}\right)_{7} \mathrm{AsO}_{2}(\mathrm{OH})_{2}\right]^{3+}+\mathrm{H}_{2} \mathrm{O}\end{array}$ & -393.12 & $\begin{array}{l}-35.5 \\
(\mathrm{M}-\mathrm{M})\end{array}$ & $\begin{array}{l}\text { Fe-As: } \\
3.28 \\
\text { Fe-Fe: } \\
3.11 \\
\text { As-O: } \\
1.68 \\
\text { As-OH: } \\
1.69 \\
1.70,1.74\end{array}$ \\
\hline $\begin{array}{l}\mathrm{Fe}^{3+} \\
\mathrm{HAsO}_{4}^{-2}\end{array}$ & $\begin{array}{l}{\left[\mathrm{Fe}_{2}(\mathrm{OH})_{2}\left(\mathrm{H}_{2} \mathrm{O}\right)_{8}\right]^{4+}+\mathrm{HAsO}_{4}{ }^{2-}} \\
\rightarrow\left[\mathrm{Fe}_{2}(\mathrm{OH})_{2}\left(\mathrm{H}_{2} \mathrm{O}\right)_{6} \mathrm{AsO}_{3}(\mathrm{OH})\right]^{2+}+2 \mathrm{H}_{2} \mathrm{O}\end{array}$ & -765.09 & $\begin{array}{l}-72.24 \\
\text { (B-B) }\end{array}$ & $\begin{array}{l}\text { Fe-As: } \\
3.00,3.10 \\
\text { Fe-Fe: } \\
3.03 \\
\text { As-O: } \\
1.66,1.74 \\
\text { As-OH: } \\
1.72\end{array}$ \\
\hline & {$\left[\mathrm{Fe}_{2}(\mathrm{OH})_{2}\left(\mathrm{H}_{2} \mathrm{O}\right)_{8}\right]^{4+}+\mathrm{HAsO}_{4}^{2-}$} & -732.71 & $\begin{array}{l}-35.87 \\
(M-B)\end{array}$ & $\begin{array}{l}\text { Fe-As: } \\
2.63 \\
\text { Fe-Fe: } \\
3.10 \\
\text { As-O: } \\
1.62 \\
1.77,1.78 \\
\text { As-OH: } \\
1.78\end{array}$ \\
\hline
\end{tabular}




\begin{tabular}{|c|c|c|c|}
\hline $\begin{array}{l}{\left[\mathrm{Fe}_{2}(\mathrm{OH})_{2}\left(\mathrm{H}_{2} \mathrm{O}\right)_{8}\right]^{4+}+\mathrm{HAsO}_{4}^{2-}} \\
\rightarrow\left[\mathrm{Fe}_{2}(\mathrm{OH})_{2}\left(\mathrm{H}_{2} \mathrm{O}\right)_{6} \mathrm{AsO}_{3}(\mathrm{OH})\right]^{2+}+2 \mathrm{H}_{2} \mathrm{O}\end{array}$ & -744.83 & $\begin{array}{l}-59.43 \\
\text { (M-B) }\end{array}$ & $\begin{array}{l}\text { Fe-As: } \\
2.71 \\
\text { Fe-Fe: } \\
3.05 \\
\text { As-O: } \\
1.61 \\
1.75,1.78 \\
\text { As-OH } \\
1.78\end{array}$ \\
\hline$\left.\rightarrow\left[\mathrm{Fe}_{2}(\mathrm{OH})_{2}\left(\mathrm{H}_{2} \mathrm{O}\right)_{8}\right]_{2}\left(\mathrm{H}_{2} \mathrm{O}\right)_{7} \mathrm{AsO}(\mathrm{OH})_{3}\right]^{2+}+\mathrm{H}_{2} \mathrm{O}$ & -776.52 & $\begin{array}{l}-65.74 \\
(M-M)\end{array}$ & $\begin{array}{l}\text { Fe-As: } \\
3.14 \\
\text { Fe-Fe: } \\
3.08 \\
\text { As-O: } \\
1.69 \\
\text { As-OH: } \\
1.70,1.72\end{array}$ \\
\hline
\end{tabular}

Scheme-S2. The optimized structures for reaction product of $\mathrm{Fe}_{2}$ oxyhydroxide dimerand $\mathrm{H}_{2} \mathrm{AsO}_{4}$ resulting inM-M boundcomplex $\left[\mathrm{Fe}_{2}(\mathrm{OH})_{2}\left(\mathrm{H}_{2} \mathrm{O}\right)_{7} \mathrm{AsO}(\mathrm{OH})_{3}\right]^{3+}$, which is converted to $\mathrm{B}-\mathrm{B}$ and $\mathrm{M}-\mathrm{B}$ products. Below the reactions are tabulated with calculated Gibb's free energies in kcal.In these structures brown balls are $\mathrm{Fe}(I I I)$, red balls are oxygen, and small greyish balls are hydrogen. 


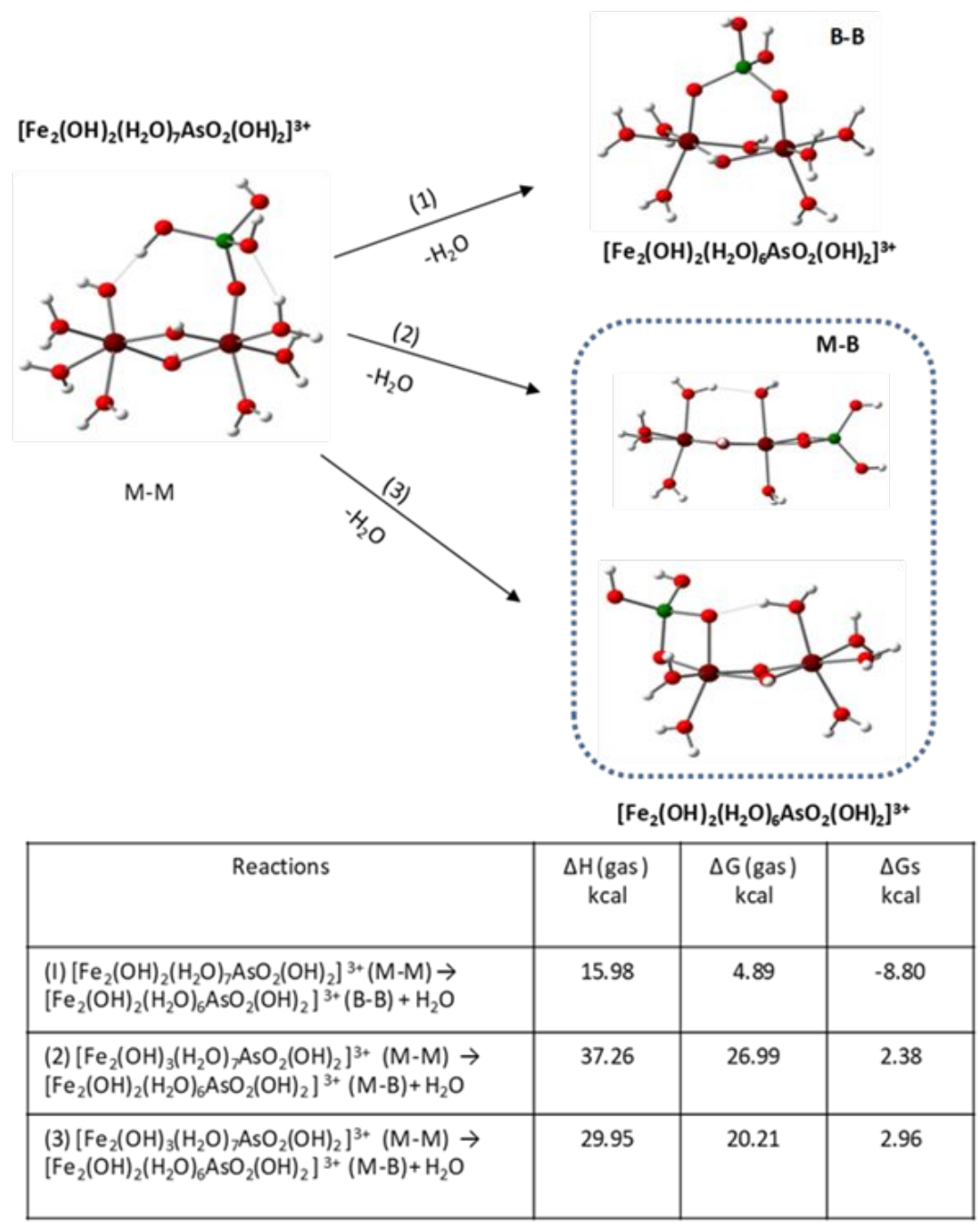

\title{
Comparison of formation energies and structural parameters using two
}

\section{different basis-sets}

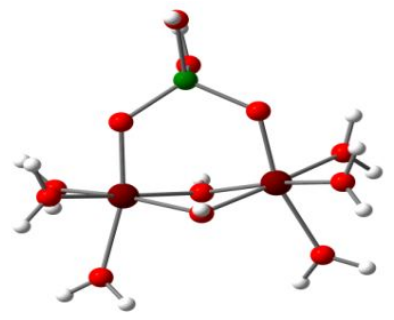

\author{
$\mathrm{H}_{2} \mathrm{AsO}_{4}$ bound to
}

$\left[\mathrm{Fe}_{2}(\mathrm{OH})_{2}\left(\mathrm{H}_{2} \mathrm{O}\right)_{8}\right]^{4+}$

Table S6. Comparison of bond distances (in A) for $\left[\mathrm{Fe}_{2}(\mathrm{OH})_{2}\left(\mathrm{H}_{2} \mathrm{O}\right)_{6}\left(\mathrm{H}_{2} \mathrm{AsO}_{4}\right)\right]^{3+}$ calculated using two basis-sets.

\begin{tabular}{|c|c|c|c|c|c|c|c|}
\hline Complex & $\mathrm{Fe}-\mathrm{OH}$ (brd) & $\begin{array}{l}\mathrm{Fe}-\mathrm{OH}_{2} \\
\text { ligands }\end{array}$ & $\begin{array}{c}O- \\
H(\text { Bridge })\end{array}$ & $\begin{array}{c}\mathrm{Fe}-\mathrm{Fe} \\
(\mathrm{via}-\mathrm{OH})\end{array}$ & $\mathrm{Fe}-\mathrm{O}$ & $A s-O$ & $A s(\mathrm{OH})$ \\
\hline $\begin{array}{l}{\left[\mathrm{Fe}_{2}(\mathrm{OH})_{2}\left(\mathrm{H}_{2} \mathrm{O}\right)_{6} \mathrm{AsO}_{2}(\mathrm{OH})_{2}\right]^{3+}} \\
\text { B3LYP/6-31G** }\end{array}$ & $1.97-2.00$ & $2.09-2.12$ & 0.97 & 3.09 & 1.90 & 1.70 & $1.7-1.72$ \\
\hline $\begin{array}{l}{\left[\mathrm{Fe}_{2}(\mathrm{OH})_{2}\left(\mathrm{H}_{2} \mathrm{O}\right)_{6} \mathrm{AsO}_{2}(\mathrm{OH})_{2}\right]^{3+}} \\
\text { B3LYP/Aug-cc-pVTZ }\end{array}$ & $1.99-2.02$ & $2.11-2.15$ & 0.97 & 3.09 & 1.92 & 1.70 & $\begin{array}{r}1.70,1.71 \\
\text { s9 }\end{array}$ \\
\hline
\end{tabular}


Table S7. Comparison of Enthalpy and Gibb's free energy in both Gas phase and solution for $\left[\mathrm{Fe}_{2}(\mathrm{OH})_{2}\left(\mathrm{H}_{2} \mathrm{O}\right)_{6} \mathrm{AsO}_{2}(\mathrm{OH})_{2}\right]^{3+}$ calculated using two basis-sets.

\begin{tabular}{|c|c|c|c|c|}
\hline $\begin{array}{l}\text { Ions } \\
\text { Interacting }\end{array}$ & Basis Set & Reactions & $\begin{array}{l}\Delta H, \Delta G \text { in } \mathrm{kcal} \\
\text { In Gas Phase }\end{array}$ & $\begin{array}{l}\Delta G_{s} \text { in } k c a l \\
\text { In solution }\end{array}$ \\
\hline $\begin{array}{l}\mathrm{Fe}^{3+} \\
\text { Dimer, } \\
\mathrm{As}^{5+}\left(\mathrm{H}_{2} \mathrm{AsO}_{4}^{-}\right)\end{array}$ & $\begin{array}{l}U B 3 L Y P / 6- \\
31 G^{* *}\end{array}$ & $\begin{array}{l}{\left[\mathrm{Fe}_{2}(\mathrm{OH})_{2}\left(\mathrm{H}_{2} \mathrm{O}\right)_{8}\right]^{4+}+\mathrm{H}_{2} \mathrm{AsO}_{4}^{-} \rightarrow} \\
{\left[\mathrm{Fe}_{2}(\mathrm{OH})_{2}\left(\mathrm{H}_{2} \mathrm{O}\right)_{6} \mathrm{AsO}_{2}(\mathrm{OH})_{2}\right]^{3+}+2 \mathrm{H}_{2} \mathrm{O}}\end{array}$ & $\begin{array}{l}-380.20 \\
-388.22\end{array}$ & -44.34 \\
\hline $\begin{array}{l}\mathrm{Fe}^{3+} \\
\text { Dimer, } \\
\mathrm{As}^{5+}\left(\mathrm{H}_{2} \mathrm{AsO}_{4}^{-}\right)\end{array}$ & $\begin{array}{l}\text { UB3LYP/Aug- } \\
c c-p V T Z\end{array}$ & $\begin{array}{l}{\left[\mathrm{Fe}_{2}(\mathrm{OH})_{2}\left(\mathrm{H}_{2} \mathrm{O}\right)_{8}\right]^{4+}+\mathrm{H}_{2} \mathrm{AsO}_{4}^{-} \rightarrow} \\
{\left[\mathrm{Fe}_{2}(\mathrm{OH})_{2}\left(\mathrm{H}_{2} \mathrm{O}\right)_{6} \mathrm{AsO}_{2}(\mathrm{OH})_{2}\right]^{3+}+2 \mathrm{H}_{2} \mathrm{O}}\end{array}$ & $\begin{array}{l}-364.36 \\
-373.03\end{array}$ & -28.49 \\
\hline
\end{tabular}

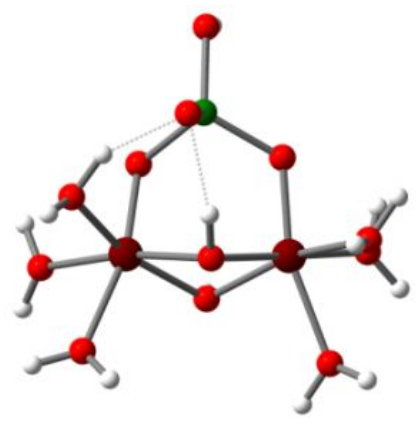

$\mathrm{HAsO}_{4}^{-2}$ bound to

$\left[\mathrm{Fe}_{2}(\mathrm{OH})_{2}\left(\mathrm{H}_{2} \mathrm{O}\right)_{8}^{4+}\right.$

Table S8. Comparison of bond distances (in A) for $\left[\mathrm{Fe}_{2}(\mathrm{OH})_{2}\left(\mathrm{H}_{2} \mathrm{O}\right)_{6} \mathrm{As} \mathrm{O}_{3}(\mathrm{OH})\right]^{2+}$ calculated using two basis-sets.

\begin{tabular}{|c|c|c|c|c|c|c|c|}
\hline Complex & $\begin{array}{l}\mathrm{Fe}-\mathrm{O} \\
\mathrm{H}(\mathrm{brd})\end{array}$ & $\begin{array}{l}\mathrm{Fe}-\mathrm{OH}_{2} \\
\text { ligands }\end{array}$ & $\begin{array}{l}\mathrm{O}- \\
\mathrm{H} \text { (Bridge) }\end{array}$ & $\begin{array}{l}\text { Fe-Fe } \\
\text { (via-OH) }\end{array}$ & $\mathrm{Fe}-\mathrm{O}$ & As-O & $\mathrm{As}(\mathrm{OH})$ \\
\hline $\begin{array}{l}{\left[\mathrm{Fe}_{2}(\mathrm{OH})_{2}\left(\mathrm{H}_{2} \mathrm{O}\right)_{6} \mathrm{AsO}_{3}(\mathrm{OH})\right]^{2+},} \\
\text { B3LYP/6-31G** }\end{array}$ & $1.98-2.03$ & $2.09-2.16$ & $0.97-0.99$ & 3.03 & 1.87 & $\begin{array}{l}1.66 \\
1.74\end{array}$ & 1.72 \\
\hline $\begin{array}{l}{\left[\mathrm{Fe}_{2}(\mathrm{OH})_{2}\left(\mathrm{H}_{2} \mathrm{O}\right)_{6} \mathrm{AsO}_{3}(\mathrm{OH})\right]^{2+},} \\
\text { B3LYP/Aug-cC-pVTZ }\end{array}$ & $1.99-2.05$ & $2.11-2.18$ & 0.97 & 3.05 & 1.88 & $\begin{array}{l}1.64- \\
1.74\end{array}$ & 1.72 \\
\hline
\end{tabular}

Table S9. Comparison of Enthalpy and Gibb's free energy in both gas phase and solution for $\left[\mathrm{Fe}_{2}(\mathrm{OH})_{2}\left(\mathrm{H}_{2} \mathrm{O}\right)_{6} \mathrm{AsO}_{3}(\mathrm{OH})\right]^{2+}$ calculated using two basis-sets.

\begin{tabular}{|l|l|l|l|l|}
\hline $\begin{array}{l}\text { lons } \\
\text { Interacting }\end{array}$ & Basis Set & Reactions & $\begin{array}{l}\Delta \mathrm{H}, \Delta \mathrm{G} \text { in } \mathrm{kcal} \\
\text { In Gas Phase }\end{array}$ & $\begin{array}{l}\Delta \mathrm{G}_{\mathrm{s}}(\mathrm{kcal}) \\
\text { In water }\end{array}$ \\
\hline
\end{tabular}




\begin{tabular}{|c|c|c|c|c|}
\hline $\begin{array}{l}\mathrm{Fe}^{3+} \\
\text { Dimer, } \\
\mathrm{As}^{5+}\left(\mathrm{H}_{2} \mathrm{AsO}_{4}{ }^{2-}\right)\end{array}$ & $\begin{array}{l}\text { UB3LYP/6- } \\
31 G^{* *}\end{array}$ & $\begin{array}{l}{\left[\mathrm{Fe}_{2}(\mathrm{OH})_{2}\left(\mathrm{H}_{2} \mathrm{O}\right)_{8}\right]^{4+}+\mathrm{HAsO}_{4}^{2-}} \\
\rightarrow\left[\mathrm{Fe}_{2}(\mathrm{OH})_{2}\left(\mathrm{H}_{2} \mathrm{O}\right)_{6} \mathrm{AsO}_{3}(\mathrm{OH})\right]^{2+}+2 \mathrm{H}_{2} \mathrm{O}\end{array}$ & $\begin{array}{l}-758.25 \\
-765.09\end{array}$ & -72.24 \\
\hline $\begin{array}{l}\mathrm{Fe}^{3+} \\
\text { Dimer, } \\
\mathrm{As}^{5+}\left(\mathrm{H}_{2} \mathrm{AsO}_{4}{ }^{2-}\right)\end{array}$ & $\begin{array}{l}\text { UB3LYP/Aug } \\
\text {-cc-pVTZ }\end{array}$ & $\begin{array}{l}{\left[\mathrm{Fe}_{2}(\mathrm{OH})_{2}\left(\mathrm{H}_{2} \mathrm{O}\right)_{8}\right]^{4+}+\mathrm{HAsO}_{4}{ }^{2-}} \\
\rightarrow\left[\mathrm{Fe}_{2}(\mathrm{OH})_{2}\left(\mathrm{H}_{2} \mathrm{O}\right)_{6} \mathrm{AsO}_{3}(\mathrm{OH})\right]^{2+}+2 \mathrm{H}_{2} \mathrm{O}\end{array}$ & $\begin{array}{l}-716.62 \\
-723.63\end{array}$ & -44.58 \\
\hline
\end{tabular}

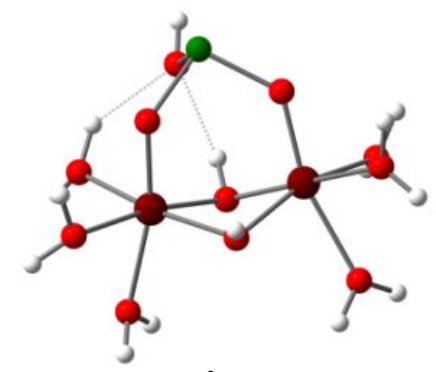

\section{$\operatorname{As}(\mathrm{OH})_{3}$ bound to}

$\left[\mathrm{Fe}_{2}(\mathrm{OH})_{2}\left(\mathrm{H}_{2} \mathrm{O}\right)_{8}\right]^{4+}$

Table S10. Comparison of bond distances (in $\mathrm{A})$ for $\left[\mathrm{Fe}_{2}(\mathrm{OH})_{2}\left(\mathrm{H}_{2} \mathrm{O}\right)_{6} \mathrm{AsO}_{2}(\mathrm{OH})\right]^{2+}$ calculated using two basissets

\begin{tabular}{|c|c|c|c|c|c|c|c|}
\hline Complex & $\begin{array}{c}\mathrm{Fe}-\mathrm{O} \\
\mathrm{H}(\mathrm{brd})\end{array}$ & $\begin{array}{l}\mathrm{Fe}-\mathrm{OH}_{2} \\
\text { ligands }\end{array}$ & $\begin{array}{c}\mathrm{O}-\mathrm{H} \\
\text { (Bridge) }\end{array}$ & $\begin{array}{c}\mathrm{Fe}-\mathrm{Fe} \\
(\mathrm{via}-\mathrm{OH})\end{array}$ & $\mathrm{Fe}-\mathrm{O}$ & $A s-O$ & $\mathrm{As}(\mathrm{OH})$ \\
\hline $\begin{array}{l}{\left[\mathrm{Fe}_{2}(\mathrm{OH})_{2}\left(\mathrm{H}_{2} \mathrm{O}\right)_{6} \mathrm{AsO}_{2}(\mathrm{OH})\right]^{2+},} \\
\text { B3LYP/6-31G** }\end{array}$ & $\begin{array}{l}2.00-2.01 \\
\text { (H-bond) }\end{array}$ & $2.12-2.17$ & $0.97-0.98$ & 2.98 & 1.83 & $\begin{array}{l}1.77, \\
1.78\end{array}$ & 1.86 \\
\hline $\begin{array}{l}{\left[\mathrm{Fe}_{2}(\mathrm{OH})_{2}\left(\mathrm{H}_{2} \mathrm{O}\right)_{6} \mathrm{AsO}_{2}(\mathrm{OH})\right]^{2+},} \\
\text { B3LYP/Aug-cc-pVTZ }\end{array}$ & $2.01-2.02$ & $2.15-2.23$ & $0.96-0.97$ & 2.99 & 1.83 & 1.79 & 1.82 \\
\hline
\end{tabular}

Table 11. Comparison of Enthalpy and Gibb's free energy in both gas phase and solutionfor $\left[\mathrm{Fe}(\mathrm{OH})_{2}\left(\mathrm{H}_{2} \mathrm{O}\right)_{6} \mathrm{AsO}_{2}(\mathrm{OH})\right]^{2+}$ calculated using two basis-sets

\begin{tabular}{|c|c|c|c|c|}
\hline $\begin{array}{c}\text { Ions } \\
\text { Interacting }\end{array}$ & Basis Set & Reactions & $\begin{array}{l}\Delta \mathrm{H}, \Delta \mathrm{G} \text { in kcal } \\
\text { In Gas Phase }\end{array}$ & $\begin{array}{c}\Delta \mathrm{G}_{\mathrm{s}} \text { in kcal } \\
\text { (water) }\end{array}$ \\
\hline $\begin{array}{l}\mathrm{Fe}^{3+} \\
\text { Dimer, } \\
\mathrm{As}^{3+}, \mathrm{As}(\mathrm{OH})_{3}\end{array}$ & $\begin{array}{l}\text { UB3LYP/6- } \\
31 G^{* *}\end{array}$ & $\begin{array}{l}{\left[\mathrm{Fe}_{2}(\mathrm{OH})_{2}\left(\mathrm{H}_{2} \mathrm{O}\right)_{8}\right]^{4+}+} \\
\mathrm{As}(\mathrm{OH})_{3} \rightarrow\left[\mathrm{Fe}_{2}(\mathrm{OH})_{2}\left(\mathrm{H}_{2} \mathrm{O}\right)_{6} \mathrm{AsO}_{2}(\mathrm{OH})\right]^{2+}+ \\
2 \mathrm{H}_{3} \mathrm{O}^{+}\end{array}$ & $\begin{array}{l}-286.72 \\
-294.15\end{array}$ & +5.90 \\
\hline $\begin{array}{l}\mathrm{Fe}^{3+} \\
\text { Dimer, } \\
\mathrm{As}^{3+}, \mathrm{As}(\mathrm{OH})_{3}\end{array}$ & $\begin{array}{l}\text { UB3LYP/Aug- } \\
\text { cc-pVTZ }\end{array}$ & $\begin{array}{l}{\left[\mathrm{Fe}_{2}(\mathrm{OH})_{2}\left(\mathrm{H}_{2} \mathrm{O}\right)_{8}\right]^{4+}+} \\
\mathrm{As}(\mathrm{OH})_{3} \rightarrow\left[\mathrm{Fe}_{2}(\mathrm{OH})_{2}\left(\mathrm{H}_{2} \mathrm{O}\right)_{6} \mathrm{AsO}_{2}(\mathrm{OH})\right]^{2+}+ \\
2 \mathrm{H}_{3} \mathrm{O}^{+}\end{array}$ & $\begin{array}{l}-273.10 \\
-282.53\end{array}$ & +21.93 \\
\hline
\end{tabular}

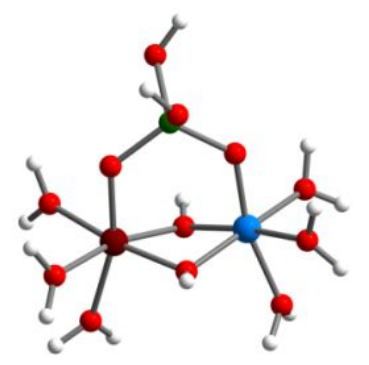

$\mathrm{H}_{2} \mathrm{AsO}_{4}$ bound to

$\left[\mathrm{FeAl}(\mathrm{OH})_{2}\left(\mathrm{H}_{2} \mathrm{O}\right)_{8}\right]^{4+}$ 
Table S12. Comparison of bond distances (in $\AA$ ) for $\left[\mathrm{FeAl}(\mathrm{OH})_{2}\left(\mathrm{H}_{2} \mathrm{O}\right)_{6} \mathrm{AsO}_{2}(\mathrm{OH})_{2}\right]^{3+}$ calculated using two basissets.

\begin{tabular}{|c|c|c|c|c|c|c|c|c|c|c|}
\hline Complex & $\begin{array}{l}\text { Fe-O } \\
\mathrm{H} \text { (brd) }\end{array}$ & $\begin{array}{c}\mathrm{Al}-\mathrm{OH} \\
\text { (brd) }\end{array}$ & $\begin{array}{l}\mathrm{Fe}-\mathrm{OH}_{2} \\
\text { ligands }\end{array}$ & $\begin{array}{l}\mathrm{Al}-\mathrm{OH}_{2} \\
\text { ligands }\end{array}$ & $\begin{array}{l}\mathrm{O}-\mathrm{H} \\
\text { (brd) }\end{array}$ & $\begin{array}{c}\text { Fe-Al } \\
\text { (via } \\
-\mathrm{OH} \text { ) }\end{array}$ & $\mathrm{Fe}-\mathrm{O}$ & Al-O & As-O & $\mathrm{As}(\mathrm{OH})$ \\
\hline $\begin{array}{l}{\left[\mathrm{FeAl}(\mathrm{OH})_{2}\left(\mathrm{H}_{2} \mathrm{O}\right)_{6} \mathrm{AsO}_{2}\right.} \\
\left.(\mathrm{OH})_{2}\right]^{3+} \\
\mathrm{B} 3 \mathrm{LYP} / 6-31 \mathrm{G} * *\end{array}$ & $\begin{array}{l}1.98- \\
2.01\end{array}$ & 1.89 & $\begin{array}{l}2.10- \\
2.13\end{array}$ & $\begin{array}{l}1.97- \\
1.99\end{array}$ & $\begin{array}{l}0.97- \\
0.98\end{array}$ & 2.99 & 1.89 & 1.83 & $\begin{array}{l}1.69 \\
1.70\end{array}$ & 1.72 \\
\hline $\begin{array}{l}{\left[\mathrm{FeAl}(\mathrm{OH})_{2}\left(\mathrm{H}_{2} \mathrm{O}\right)_{6} \mathrm{AsO}_{2}\right.} \\
\left.(\mathrm{OH})_{2}\right]^{3+} \\
\text { B3LYP/Aug-cc-pVTZ }\end{array}$ & $\begin{array}{l}2.00 \\
2.02\end{array}$ & $\begin{array}{l}1.88, \\
1.89\end{array}$ & $\begin{array}{l}2.11- \\
2.15\end{array}$ & $\begin{array}{l}1.97- \\
1.99\end{array}$ & 0.96 & 3.00 & 1.91 & 1.84 & $\begin{array}{l}1.68, \\
1.69\end{array}$ & 1.71 \\
\hline
\end{tabular}

Table S13. Comparison of Enthalpy and Gibb's free energy in both Gas phase and solution for $\left[\mathrm{FeAl}(\mathrm{OH})_{2}\left(\mathrm{H}_{2} \mathrm{O}\right)_{6} \mathrm{AsO} \mathrm{O}_{2}(\mathrm{OH})_{2}\right]^{3+}$ calculated using two basis-sets

\begin{tabular}{|l|l|l|l|l|}
\hline $\begin{array}{l}\text { lons } \\
\text { Interacting }\end{array}$ & Basis Set & Reactions & $\begin{array}{l}\Delta \mathrm{H}, \Delta \mathrm{G} \text { in kcal } \\
\text { In Gas Phase }\end{array}$ & $\begin{array}{l}\Delta \mathrm{G}_{\mathrm{s}} \text { in kcal } \\
\text { (water) }\end{array}$ \\
\hline $\begin{array}{l}\mathrm{Fe}^{3+}, \mathrm{Al}^{3+} \text { Dimer, } \\
\mathrm{As}^{5+}\left(\mathrm{H}_{2} \mathrm{AsO}_{4}^{-}\right)\end{array}$ & UB3LYP/6-31G** & $\begin{array}{l}{\left[\mathrm{FeAl}(\mathrm{OH})_{2}\left(\mathrm{H}_{2} \mathrm{O}\right)_{8}\right]^{4+}+\mathrm{H}_{2} \mathrm{AsO}_{4}^{-}} \\
\rightarrow\left[\mathrm{FeAl}(\mathrm{OH})_{2}\left(\mathrm{H}_{2} \mathrm{O}\right)_{6} \mathrm{AsO}_{2}(\mathrm{OH})_{2}\right]^{3+}+2 \mathrm{H}_{2} \mathrm{O}\end{array}$ & -380.41 \\
\hline $\begin{array}{l}\mathrm{Fe}^{3+}, \mathrm{Al}^{3+} \text { Dimer, } \\
\mathrm{As}^{5+}\left(\mathrm{H}_{2} \mathrm{AsO}_{4}^{-}\right)\end{array}$ & UB3LYP/Aug-cc- & $\begin{array}{l}{\left[\mathrm{FeAl}(\mathrm{OH})_{2}\left(\mathrm{H}_{2} \mathrm{O}\right)_{8}\right]^{4+}+\mathrm{H}_{2} \mathrm{AsO}_{4}^{-}} \\
\end{array}$ & $\rightarrow\left[\mathrm{FeAl}(\mathrm{OH})_{2}\left(\mathrm{H}_{2} \mathrm{O}\right)_{6} \mathrm{AsO}_{2}(\mathrm{OH})_{2}\right]^{3+}+2 \mathrm{H}_{2} \mathrm{O}$ & -44.15 \\
\hline
\end{tabular}

Table S14.

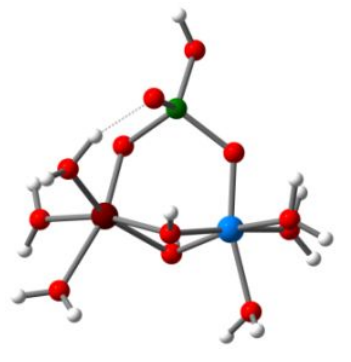

$\mathrm{HAsO}_{4}{ }^{2-}$ bound to

$\left[\mathrm{FeAl}(\mathrm{OH})_{2}\left(\mathrm{H}_{2} \mathrm{O}\right)_{8}\right]^{4+}$

Comparison of bond distances (in $A$ ) for

$\left[\mathrm{FeAl}(\mathrm{OH})_{2}\left(\mathrm{H}_{2} \mathrm{O}\right)_{6} \mathrm{AsO}_{3}(\mathrm{OH})\right]^{2+}$ calculated using two basis-sets.

\begin{tabular}{|c|c|c|c|c|c|c|c|c|c|c|}
\hline Complex & $\begin{array}{c}\mathrm{Fe}-\mathrm{O} \\
\mathrm{H} \\
(\mathrm{brd})\end{array}$ & $\begin{array}{l}\text { Al-O } \\
\mathrm{H}(\mathrm{brd})\end{array}$ & $\begin{array}{l}\mathrm{Fe}-\mathrm{OH}_{2} \\
\text { ligands }\end{array}$ & $\begin{array}{l}\mathrm{Al}-\mathrm{OH}_{2} \\
\text { ligands }\end{array}$ & $\begin{array}{c}\mathrm{O}-\mathrm{H} \\
\text { (Bridge) }\end{array}$ & $\begin{array}{c}\text { Fe-Al } \\
\text { (via - } \\
\mathrm{OH} \text { ) }\end{array}$ & $\mathrm{Fe}-\mathrm{O}$ & $\mathrm{Al}-\mathrm{O}$ & As-O & $\begin{array}{c}\mathrm{As}(\mathrm{O} \\
\mathrm{H})\end{array}$ \\
\hline $\begin{array}{l}{\left[\mathrm{FeAl}(\mathrm{OH})_{2}\left(\mathrm{H}_{2} \mathrm{O}\right)_{6} \mathrm{AsO}_{3}\right.} \\
(\mathrm{OH})]^{2+}, \mathrm{B} 3 \mathrm{LYP} / 6-31 \mathrm{G}^{* *}\end{array}$ & $\begin{array}{l}2.03 \\
2.06\end{array}$ & $\begin{array}{l}1.87 \\
1.89\end{array}$ & $\begin{array}{l}2.07- \\
2.14\end{array}$ & $\begin{array}{l}1.98, \\
2.01\end{array}$ & 0.97 & 2.96 & 1.86 & 1.81 & $\begin{array}{l}1.66- \\
1.74\end{array}$ & 1.72 \\
\hline $\begin{array}{l}{\left[\mathrm{FeAl}(\mathrm{OH})_{2}\left(\mathrm{H}_{2} \mathrm{O}\right)_{6} \mathrm{AsO}_{3}\right.} \\
(\mathrm{OH})]^{2+}, \mathrm{B} 3 \mathrm{LYP} / \text { Aug-cc-PVTZ }\end{array}$ & $\begin{array}{l}2.05, \\
2.09\end{array}$ & 1.87 & $\begin{array}{l}2.09- \\
2.17\end{array}$ & $\begin{array}{l}1.98- \\
2.01\end{array}$ & 0.96 & 2.97 & 1.86 & 1.82 & $\begin{array}{l}1.65- \\
1.74\end{array}$ & 1.72 \\
\hline
\end{tabular}

Table S15. Comparison of Enthalpy and Gibb's free energy in both Gas phase and solution for $\left[\mathrm{FeAl}(\mathrm{OH})_{2}\left(\mathrm{H}_{2} \mathrm{O}\right)_{6} \mathrm{AsO}_{3}(\mathrm{OH})\right]^{2+}$ calculated using two basis-sets. 


\begin{tabular}{|c|c|c|c|c|}
\hline $\begin{array}{c}\text { lons } \\
\text { Interacting }\end{array}$ & Basis Set & Reactions & $\begin{array}{l}\Delta \mathrm{H}, \Delta \mathrm{G} \text { in } \\
\text { kcal In Gas } \\
\text { Phase }\end{array}$ & $\begin{array}{l}\Delta \mathrm{G}_{\mathrm{s}} \text { in } \mathrm{kcal} \\
\text { In water }\end{array}$ \\
\hline $\begin{array}{l}\mathrm{Fe}^{3+}, \mathrm{Al}^{3+} \text { Dimer, } \\
\mathrm{As}^{5+}\left(\mathrm{HAsO}_{4}^{2-}\right)\end{array}$ & UB3LYP/6-31G** & $\begin{array}{l}{\left[\mathrm{FeAl}(\mathrm{OH})_{2}\left(\mathrm{H}_{2} \mathrm{O}\right)_{8}\right]^{4+}+\mathrm{HAsO}_{4}{ }^{2-}} \\
\rightarrow\left[\mathrm{FeAl}(\mathrm{OH})_{2}\left(\mathrm{H}_{2} \mathrm{O}\right)_{6} \mathrm{AsO}_{3}(\mathrm{OH})\right]^{2+}+2 \mathrm{H}_{2} \mathrm{O}\end{array}$ & $\begin{array}{l}-757.79 \\
-764.49\end{array}$ & -69.95 \\
\hline $\begin{array}{l}\mathrm{Fe}^{3+}, \mathrm{Al}^{3+} \text { Dimer, } \\
\mathrm{As}^{5+}\left(\mathrm{HAsO}_{4}^{2-}\right)\end{array}$ & $\begin{array}{l}\text { UB3LYP/Aug-cc- } \\
\text { pVTZ }\end{array}$ & $\begin{array}{l}{\left[\mathrm{FeAl}(\mathrm{OH})_{2}\left(\mathrm{H}_{2} \mathrm{O}\right)_{8}\right]^{4+}+\mathrm{HAsO}_{4}{ }^{2-}} \\
\rightarrow\left[\mathrm{FeAl}(\mathrm{OH})_{2}\left(\mathrm{H}_{2} \mathrm{O}\right)_{6} \mathrm{AsO}_{3}(\mathrm{OH})\right]^{2+}+2 \mathrm{H}_{2} \mathrm{O}\end{array}$ & $\begin{array}{l}-716.01 \\
-722.98\end{array}$ & -41.01 \\
\hline
\end{tabular}

Table S16. Comparison of bond distances (in $A)$ for $\left[\mathrm{FeAl}(\mathrm{OH})_{2}\left(\mathrm{H}_{2} \mathrm{O}\right)_{6} \mathrm{AsO}_{2}(\mathrm{OH})\right]^{2+}$ calculated using two basissets

Table S17. Comparison of Enthalpy and Gibb's free energy in both Gas phase and solution for $\left[\mathrm{FeAl}(\mathrm{OH})_{2}\left(\mathrm{H}_{2} \mathrm{O}\right)_{6} \mathrm{AsO}_{2}(\mathrm{OH})\right]^{2+}$ calculated using two basis-sets.

\begin{tabular}{|c|c|c|c|c|c|c|c|c|c|c|}
\hline Complex & $\begin{array}{c}\text { Fe-O } \\
\mathrm{H} \text { (brd) }\end{array}$ & $\begin{array}{c}\mathrm{Al}-\mathrm{O} \\
\mathrm{H} \\
\text { (brd) }\end{array}$ & $\begin{array}{l}\text { Fe-OH } \\
\text { ligands }\end{array}$ & $\begin{array}{l}\mathrm{Al}-\mathrm{OH}_{2} \\
\text { ligands }\end{array}$ & $\begin{array}{l}\mathrm{O}-\mathrm{H} \\
\text { (Brd) }\end{array}$ & $\begin{array}{c}\text { Fe-Al } \\
\text { (via - } \\
\text { OH) }\end{array}$ & $\begin{array}{c}\mathrm{Fe}- \\
\mathrm{O}\end{array}$ & Al-O & As-O & $\mathrm{As}(\mathrm{OH})$ \\
\hline $\begin{array}{l}{\left[\mathrm{FeAl}(\mathrm{OH})_{2}\left(\mathrm{H}_{2} \mathrm{O}\right)_{6} \mathrm{AsO}_{2}(\mathrm{OH})\right]^{2+}} \\
\mathrm{B} 3 \mathrm{LYP} / 6-31 \mathrm{G} * *\end{array}$ & $\begin{array}{l}2.01- \\
2.03\end{array}$ & 1.89 & $2.13-2.18$ & $1.99-2.01$ & $\begin{array}{l}0.97- \\
0.98\end{array}$ & 2.89 & 1.81 & 1.80 & $\begin{array}{l}1.74- \\
1.81\end{array}$ & 1.88 \\
\hline $\begin{array}{l}{\left[\mathrm{FeAl}(\mathrm{OH})_{2}\left(\mathrm{H}_{2} \mathrm{O}\right)_{6} \mathrm{AsO}_{2}(\mathrm{OH})\right]^{2+}} \\
\text { B3LYP/Aug-cc-Pvtz }\end{array}$ & $\begin{array}{l}2.03- \\
2.04\end{array}$ & 1.88 & $2.15-2.22$ & $1.99-2.01$ & $\begin{array}{l}0.96- \\
0.97\end{array}$ & 2.92 & 1.82 & 1.80 & $\begin{array}{l}1.74- \\
1.82\end{array}$ & 1.86 \\
\hline
\end{tabular}

\section{Gibb's free energies for $\mathrm{As}^{3+} / \mathrm{As}^{5+}$ binding to tertramericFe(III) clusters}

\begin{tabular}{|l|l|l|l|l|}
\hline $\begin{array}{l}\text { lons } \\
\text { Interacting }\end{array}$ & Basis Set & Reactions & $\begin{array}{l}\Delta \mathrm{H}, \Delta \mathrm{G} \text { in } \\
\text { kcal } \\
\text { In Gas Phase }\end{array}$ & $\begin{array}{l}\Delta \mathrm{G}_{\mathrm{s}} \text { in } \mathrm{kcal} \\
\text { In water }\end{array}$ \\
\hline $\begin{array}{l}\mathrm{Fe}^{3+}, \mathrm{Al}^{3+} \mathrm{Dimer}, \\
\mathrm{As}^{3+}, \mathrm{As}(\mathrm{OH})_{3}\end{array}$ & $\begin{array}{l}\mathrm{UB} 3 \mathrm{LYP} / \\
6-31 \mathrm{G} * *\end{array}$ & $\begin{array}{l}{\left[\mathrm{FeAl}(\mathrm{OH})_{2}\left(\mathrm{H}_{2} \mathrm{O}\right)_{8}\right]^{4+}} \\
\mathrm{As}(\mathrm{OH})_{3} \rightarrow\left[\mathrm{FeAl}(\mathrm{OH})_{2}\left(\mathrm{H}_{2} \mathrm{O}\right)_{6} \mathrm{AsO}_{2}(\mathrm{OH})\right]^{2+}+2 \mathrm{H}_{3} \mathrm{O}^{+}\end{array}$ & $\begin{array}{l}-284.26 \\
-290.87\end{array}$ & +8.09 \\
\hline $\begin{array}{l}\mathrm{Fe}^{3+}, \mathrm{Al}{ }^{3+} \mathrm{Dimer}, \\
\mathrm{As}^{3+}, \mathrm{As}(\mathrm{OH})_{3}\end{array}$ & $\begin{array}{l}\text { UB3LYP/ } \\
\text { Aug-cc- } \\
\text { pVTZ }\end{array}$ & $\begin{array}{l}{\left[\mathrm{FeAl}(\mathrm{OH})_{2}\left(\mathrm{H}_{2} \mathrm{O}\right)_{8}\right]^{4+}+} \\
\mathrm{As}(\mathrm{OH})_{3} \rightarrow\left[\mathrm{FeAl}(\mathrm{OH})_{2}\left(\mathrm{H}_{2} \mathrm{O}\right)_{6} \mathrm{AsO}_{2}(\mathrm{OH})\right]^{2+}+2 \mathrm{H}_{3} \mathrm{O}^{+}\end{array}$ & $\begin{array}{l}-268.97 \\
-276.83\end{array}$ & +26.25 \\
\hline
\end{tabular}

\section{(doped/undoped)}

Table S18.The reactions of $\mathrm{Fe}(\mathrm{III})$ oxyhydroxide tetrameric clusters with $\mathrm{As}(\mathrm{OH})_{3}$ in gas phase and in water are shown for few selected cases. Some different complexation cases like bidentate-binuclear (B-B) and 
bidentate mononuclear (M-B) type are tabulated. In all cases $\mathrm{Fe}^{3+}$ complexes are high-spin. The reaction free energies in aqueous medium are calculated form solvation free energies and corrected for standard states. ${ }^{17}$

\begin{tabular}{|c|c|c|c|c|}
\hline $\begin{array}{c}\text { Ions } \\
\text { Interacting }\end{array}$ & Reactions & $\begin{array}{c}\Delta \mathrm{H}, \Delta \mathrm{G} \\
\text { (in kcal) } \\
\text { In Gas phase }\end{array}$ & $\begin{array}{c}\Delta \mathrm{G}_{\mathrm{s}} \\
\text { (water) } \\
\text { (in kcal) }\end{array}$ & $\begin{array}{c}\text { Bond } \\
\text { Distances } \\
\text { (in } \AA \text { ) }\end{array}$ \\
\hline $\begin{array}{l}\mathrm{Fe}^{3+} \text { Tetramer } \\
\mathrm{As}^{3+},\left(\mathrm{As}(\mathrm{OH})_{3}\right.\end{array}$ & $\begin{array}{l}\left.\mathrm{Fe}_{4} \mathrm{O}_{2}(\mathrm{OH})_{4}\left(\mathrm{H}_{2} \mathrm{O}\right)_{10}\right]^{4+}+ \\
\mathrm{As}(\mathrm{OH})_{3} \rightarrow\left[\mathrm{Fe}_{4} \mathrm{O}_{2}(\mathrm{OH})_{4}\left(\mathrm{H}_{2} \mathrm{O}\right)_{8} \mathrm{AsO}_{2}(\mathrm{OH})\right]^{2+}+ \\
2 \mathrm{Fe}_{3} \mathrm{O}^{+} \\
\mathrm{As}(\mathrm{OH})_{3} \rightarrow\left[\mathrm{Fe}_{4} \mathrm{O}_{2}(\mathrm{OH})_{4}\left(\mathrm{H}_{2} \mathrm{O}\right)_{8} \mathrm{AsO}_{2}(\mathrm{OH})\right]^{2+}+ \\
2 \mathrm{H}_{3} \mathrm{O}^{+}\end{array}$ & $\begin{array}{l}\Delta H=-177.06 \\
\Delta G=-180.46\end{array}$ & $\begin{array}{l}21.88 \\
(\mathrm{M}-\mathrm{B})\end{array}$ & $\begin{array}{l}\text { Fe-As: } \\
2.71 \\
\text { Fe-Fe: } \\
2.95 \\
\text { As-O: } \\
1.79 \\
\text { As-OH: } \\
1.84 \\
\\
\text { Fe-As: } \\
3.19,3.25 \\
\text { Fe-Fe:2.88 } \\
\text { As-O:1.76 } \\
\text { As- } \\
\text { OH:1.88 }\end{array}$ \\
\hline $\begin{array}{l}\mathrm{Fe}^{3+} \text { Tetramer } \\
\mathrm{Fe}_{4} \mathrm{O}(\mathrm{OH})_{3}\left(\mathrm{H}_{2} \mathrm{O}\right)_{9}{ }^{4+} \\
\text { doped with one } \\
\mathrm{Al}^{3+} \text { ion, } \\
\mathrm{As}^{3+},\left(\mathrm{As}(\mathrm{OH})_{3}\right.\end{array}$ & $\begin{array}{l}{\left[\mathrm{Fe}_{3} \mathrm{AlO}_{2}(\mathrm{OH})_{4}\left(\mathrm{H}_{2} \mathrm{O}\right)_{10}\right]^{4+}+} \\
\mathrm{As}(\mathrm{OH})_{3} \rightarrow\left[\mathrm{Fe}_{3} \mathrm{AlO}_{2}(\mathrm{OH})_{4}\left(\mathrm{H}_{2} \mathrm{O}\right)_{8} \mathrm{AsO}_{2}(\mathrm{OH})\right]^{2+}+ \\
2 \mathrm{H}_{3} \mathrm{O}^{+} \text {(Fe center) }\end{array}$ & $\begin{array}{l}\Delta H=-174.63 \\
\Delta G=-177.51\end{array}$ & $\begin{array}{l}22.51 \\
(\mathrm{M}-\mathrm{B})\end{array}$ & $\begin{array}{l}\text { Fe-As:2.71 } \\
\text { Fe-Fe:2.96 } \\
\text { Fe-Al: } 2.89 \\
\text { As-O:1.79 } \\
\text { As- } \\
\mathrm{OH}: 1.84\end{array}$ \\
\hline & $\begin{array}{l}{\left[\mathrm{Fe}_{3} \mathrm{AlO}_{2}(\mathrm{OH})_{4}\left(\mathrm{H}_{2} \mathrm{O}\right)_{10}\right]^{4+}+} \\
\mathrm{As}(\mathrm{OH})_{3} \rightarrow\left[\mathrm{Fe}_{3} \mathrm{AlO}_{2}(\mathrm{OH})_{4}\left(\mathrm{H}_{2} \mathrm{O}\right)_{8} \mathrm{AsO}_{2}(\mathrm{OH})\right]^{2+}+ \\
2 \mathrm{H}_{3} \mathrm{O}^{+}(\mathrm{Al} \text { center })\end{array}$ & $\begin{array}{l}\Delta H=-180.37 \\
\Delta G=-185.63\end{array}$ & $\begin{array}{l}28.69 \\
(M-B)\end{array}$ & $\begin{array}{l}\text { Al-As:2.78 } \\
\text { Fe-Fe:2.93 } \\
\text { Fe-Al:2.89 } \\
\text { As-O:1.73 } \\
\text { As- } \\
\text { OH:1.82, } \\
1.89\end{array}$ \\
\hline & $\begin{array}{l}{\left[\mathrm{Fe}_{3} \mathrm{AlO}_{2}(\mathrm{OH})_{4}\left(\mathrm{H}_{2} \mathrm{O}\right)_{10}\right]^{4+}+} \\
\mathrm{As}(\mathrm{OH})_{3} \rightarrow\left[\mathrm{Fe}_{3} \mathrm{AlO}_{2}(\mathrm{OH})_{4}\left(\mathrm{H}_{2} \mathrm{O}\right)_{8} \mathrm{AsO}_{2}(\mathrm{OH})\right]^{2+}+ \\
2 \mathrm{H}_{3} \mathrm{O}^{+}\end{array}$ & $\begin{array}{l}\Delta H=-183.64 \\
\Delta G=-189.10\end{array}$ & $\begin{array}{l}23.12 \\
\text { (B-B) }\end{array}$ & $\begin{array}{l}\text { Fe-As:3.23 } \\
\text { Al-As:3.18 } \\
\text { Fe-Fe:2.91 } \\
\text { Fe-Al:2.80 }\end{array}$ \\
\hline
\end{tabular}




\begin{tabular}{|c|c|c|c|c|}
\hline & & & & $\begin{array}{l}\text { As-O: 1.76, } \\
1.80 \\
\text { As- } \\
\mathrm{OH}: 1.85\end{array}$ \\
\hline $\begin{array}{l}\mathrm{Fe}^{3+} \text { Tetramer } \\
\mathrm{Fe}_{4} \mathrm{O}(\mathrm{OH})_{3}\left(\mathrm{H}_{2} \mathrm{O}\right)_{9}{ }^{4+} \mathrm{do} \\
\text { ped with two } \mathrm{Al}^{3+} \text { ion } \\
\text { (no } \mathrm{Al}-\mathrm{OH}-\mathrm{Al} \text { bond), } \\
\mathrm{As}^{3+},\left(\mathrm{As}(\mathrm{OH})_{3}\right.\end{array}$ & 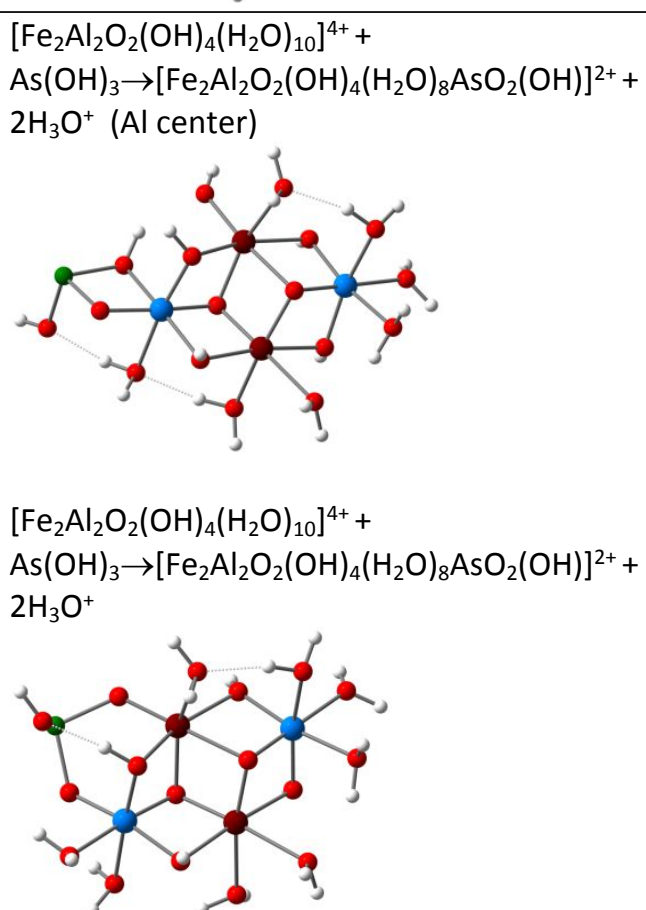 & $\begin{array}{l}\Delta \mathrm{H}=-180.63 \\
\Delta \mathrm{G}=-184.80 \\
\\
\Delta \mathrm{H}=-183.70 \\
\Delta \mathrm{G}=-188.61\end{array}$ & $\begin{array}{l}26.18 \\
\text { (M-B) }\end{array}$ & $\begin{array}{l}\text { Al-As:2.78 } \\
\text { Fe-Fe:2.89 } \\
\text { Fe-Al:2.84- } \\
2.89 \\
\text { Al-Al:5.00 } \\
\text { As-O:1.73 } \\
\text { As- } \\
\text { OH:1.82, } \\
1.89 \\
\\
\text { Fe-As:3.23 } \\
\text { Fe-Fe:2.89 } \\
\text { Al-As:3.18 } \\
\text { Al-Al:4.96 } \\
\text { Fe-Al:2.81- } \\
2.97 \\
\text { As-O:1.76, } \\
1.80 \\
\text { As- } \\
\text { OH:1.85 }\end{array}$ \\
\hline $\begin{array}{l}\mathrm{Fe}^{3+} \mathrm{Tetramer} \\
\mathrm{Fe}_{4} \mathrm{O}(\mathrm{OH})_{3}\left(\mathrm{H}_{2} \mathrm{O}\right)_{9}{ }^{4+} \text { do } \\
\text { ped with two } \\
\mathrm{Al}^{3+} \text { ions }(\mathrm{Al}-\mathrm{OH}-\mathrm{Al} \\
\text { bond present }) \\
\mathrm{As}^{3+}, \mathrm{As}(\mathrm{OH})_{3}\end{array}$ & 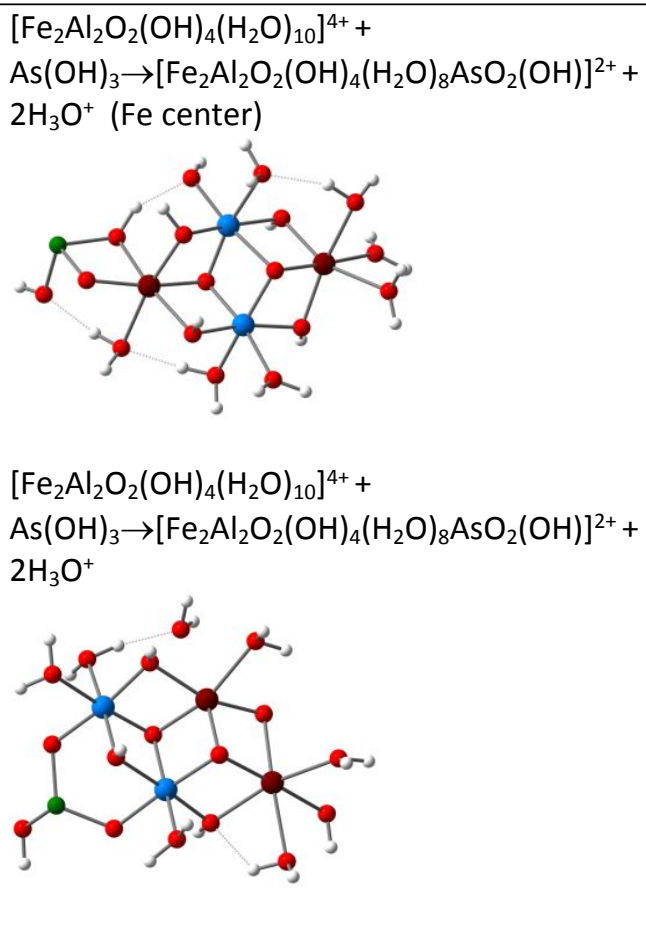 & $\begin{array}{l}\Delta \mathrm{HH}=-178.96 \\
\Delta \mathrm{G}=-183.56 \\
\Delta \mathrm{H}=-159.87 \\
\Delta \mathrm{G}=-168.94\end{array}$ & $\begin{array}{l}20.74 \\
\text { (M-B) } \\
\\
\\
\\
\\
\\
44.84 \\
\text { (B-B) }\end{array}$ & $\begin{array}{l}\text { Fe-As:2.85 } \\
\text { Fe-Fe:4.99 } \\
\text { Al-Al:2.83 } \\
\text { Fe-Al:2.83- } \\
2.90 \\
\text { As-0:1.75 } \\
\text { As- } \\
\text { OH:1.82, } \\
1.86 \\
\\
\text { Fe-As: } \\
\text { 5.07, } 5.98 \\
\text { Fe-Fe:2.92 } \\
\text { Al-As:3.03, } \\
3.09 \\
\text { Al-Al:2.85 } \\
\text { Fe-Al:2.74, } \\
2.99 \\
\text { As-0:1.71 } \\
\text { As- } \\
\text { OH:1.76 }\end{array}$ \\
\hline
\end{tabular}




\begin{tabular}{|c|c|c|c|c|}
\hline $\begin{array}{l}\mathrm{Fe}^{3+} \text { Tetramer } \\
\mathrm{Fe}_{4} \mathrm{O}(\mathrm{OH})_{3}\left(\mathrm{H}_{2} \mathrm{O}\right)_{9}{ }^{4+} \mathrm{do} \\
\text { ped with three } \mathrm{Al}^{3+} \\
\text { ion } \mathrm{As}^{3+}, \mathrm{As}(\mathrm{OH})_{3}\end{array}$ & $\begin{array}{l}{\left[\mathrm{FeAl}_{3} \mathrm{O}_{2}(\mathrm{OH})_{4}\left(\mathrm{H}_{2} \mathrm{O}\right)_{10}\right]^{4+}+} \\
\mathrm{As}(\mathrm{OH})_{3} \rightarrow\left[\mathrm{FeAl}_{3} \mathrm{O}_{2}(\mathrm{OH})_{4}\left(\mathrm{H}_{2} \mathrm{O}\right)_{8} \mathrm{AsO}_{2}(\mathrm{OH})\right]^{2+}+ \\
2 \mathrm{H}_{3} \mathrm{O}^{+}\end{array}$ & $\begin{array}{l}\Delta \mathrm{H}=-179.96 \\
\Delta \mathrm{G}=-183.20\end{array}$ & $\begin{array}{l}26.52 \\
\text { (B-B) }\end{array}$ & $\begin{array}{l}\text { Fe-As:3.14 } \\
\text { Al-As:3.19 } \\
\text { Al-Al:2.76, } \\
2.82 \\
\text { Fe-Al:2.77, } \\
2.89 \\
\text { As-O:1.76, } \\
1.79 \\
\text { As- } \\
\text { OH:1.87 }\end{array}$ \\
\hline
\end{tabular}

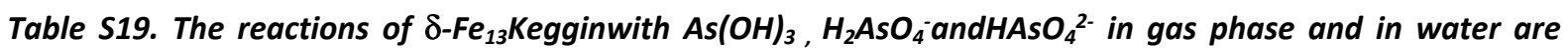
shown for few selected cases. For each complexation cases, both the bidentate-binuclear (B-B) and bidentate mononuclear (M-B) and also mononuclear-mondentate (M-M) type had been tabulated. Some variations in $M-B$ cases are also include. In all cases, $\mathrm{Fe}^{3+}$ complexes are high-spin. The reaction free energies in aqueous medium are calculated form solvation free energies and corrected for standard states. ${ }^{17}$

\begin{tabular}{|c|c|c|c|c|}
\hline \multicolumn{5}{|c|}{ Reactions of $\mathrm{Fe}_{13} \mathrm{Keggin}$ ion withAs $(\mathrm{OH})_{3}, \mathrm{HAsO}_{4}{ }^{2-}, \mathrm{H}_{2} \mathrm{AsO}_{4}{ }^{-}$} \\
\hline $\begin{array}{c}\text { Ions } \\
\text { Interacting }\end{array}$ & Reactions & $\begin{array}{l}\Delta \mathrm{H}, \Delta \mathrm{G} \\
\text { ingas- } \\
\text { phase } \\
\text { (kcal) }\end{array}$ & $\begin{array}{c}\Delta \mathrm{G}_{\mathrm{s}} \text { in water } \\
\text { (in kcal) }\end{array}$ & $\begin{array}{c}\text { Bond } \\
\text { Distance } \\
\text { (in } \AA \text { ) }\end{array}$ \\
\hline $\begin{array}{l}\delta \text { - } \mathrm{Fe}_{13} \text { Keggin, } \\
\mathrm{As}^{3+}\left(\mathrm{As}(\mathrm{OH})_{3}\right) \\
\text { binding }\end{array}$ & $\begin{array}{l}{\left[\mathrm{Fe}_{13} \mathrm{O}_{4}(\mathrm{OH})_{24}\left(\mathrm{H}_{2} \mathrm{O}\right)_{12}\right]^{]_{+}+}} \\
\mathrm{As}(\mathrm{OH})_{3} \rightarrow\left[\mathrm{Fe}_{13} \mathrm{O}_{4}(\mathrm{OH})_{24}\left(\mathrm{H}_{2} \mathrm{O}\right)_{10} \mathrm{AsO}_{2}(\mathrm{OH})\right]^{5+}+ \\
2 \mathrm{H}_{3} \mathrm{O}^{+}\end{array}$ & $\begin{array}{l}-380.23 \\
-388.39\end{array}$ & $\begin{array}{l}25.9 \\
\text { (B-B) } \\
\text { (non } \\
\text { protonated) }\end{array}$ & $\begin{array}{l}\text { Fe-As } \\
3.25,3.26 \\
\text { Fe-Fe } \\
3.16 \\
\text { As-O } \\
1.72,1.73 \\
\text { As-OH } \\
1.75\end{array}$ \\
\hline
\end{tabular}




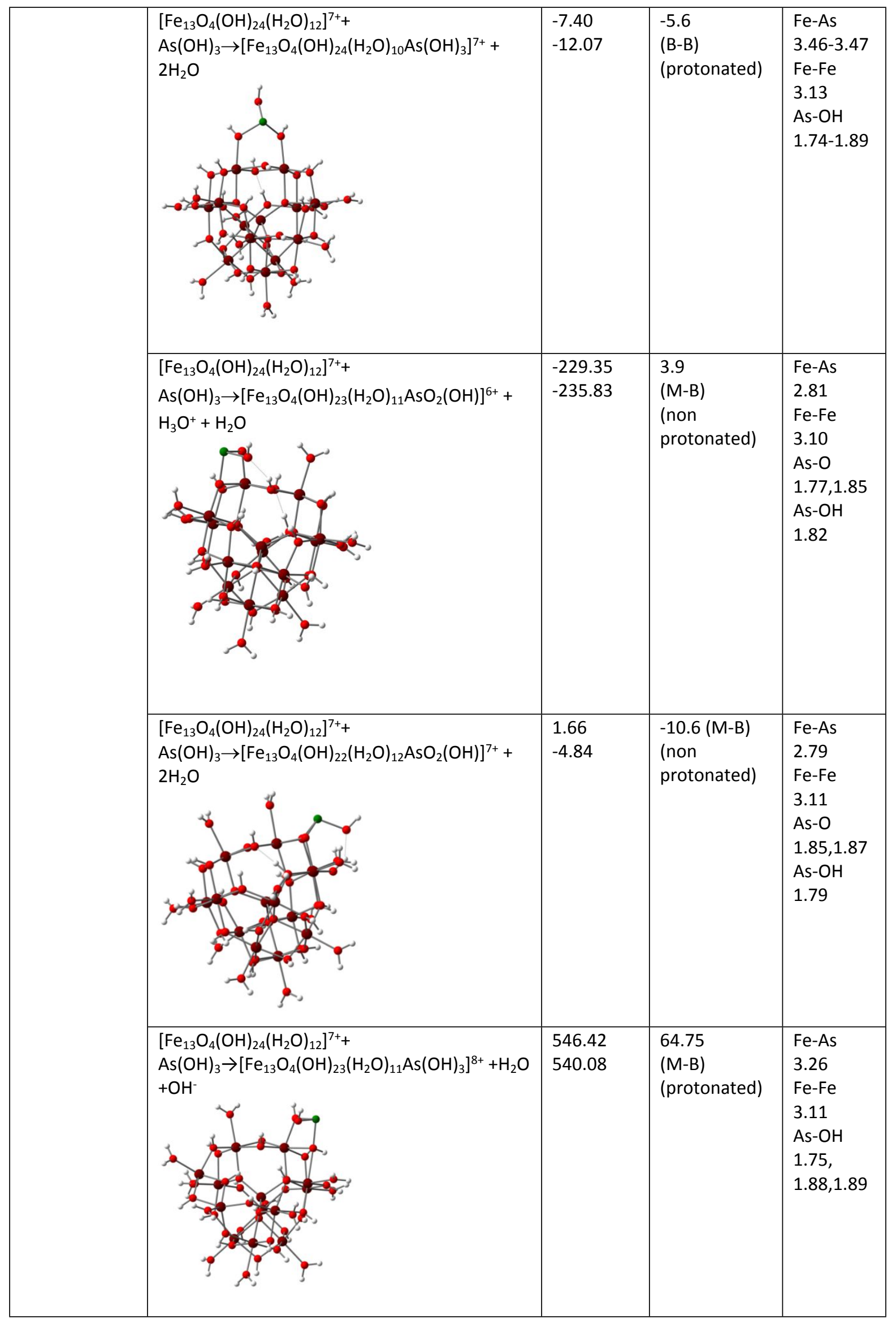




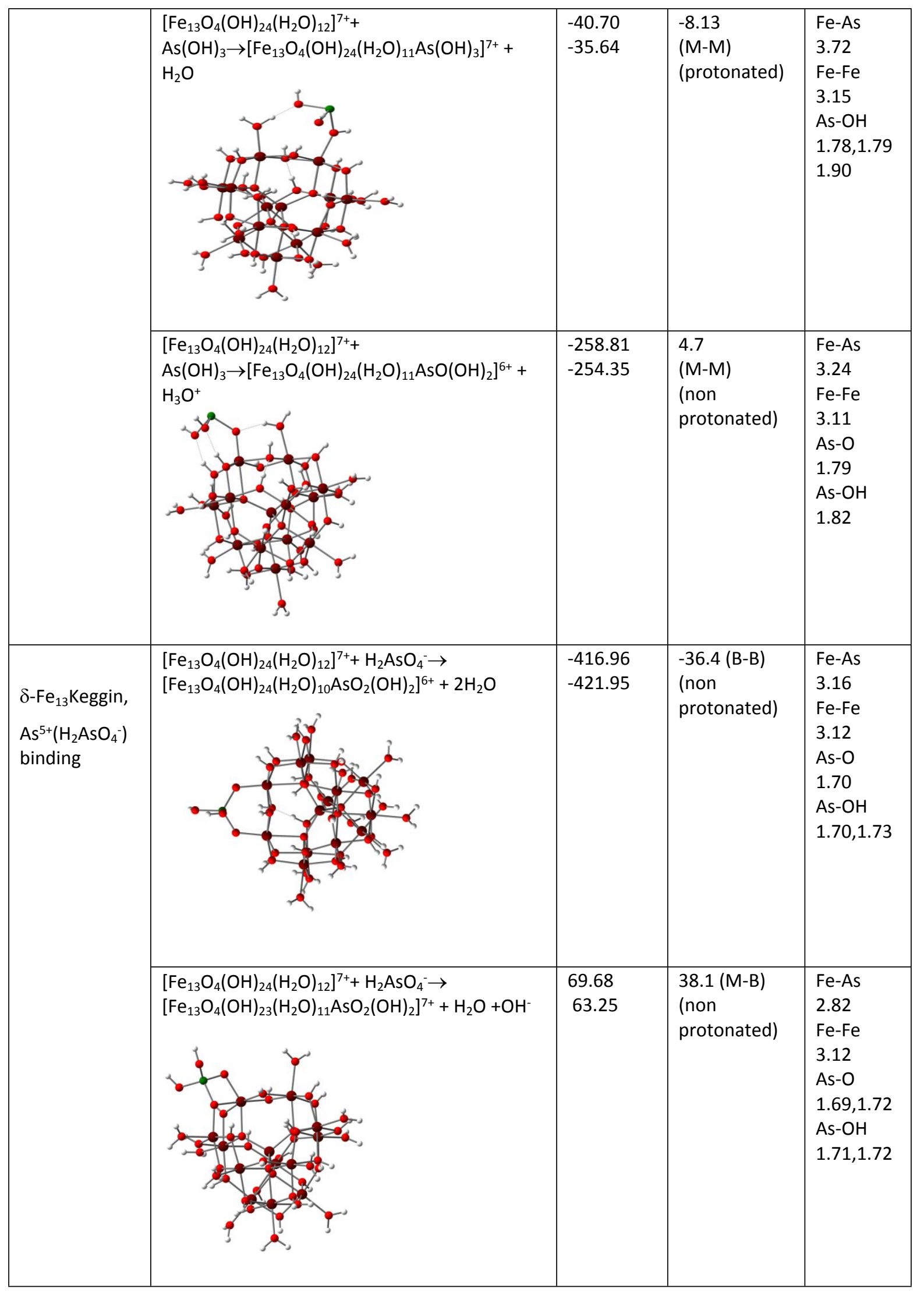




\begin{tabular}{|c|c|c|c|c|}
\hline & {$\left[\mathrm{Fe}_{13} \mathrm{O}_{4}(\mathrm{OH})_{24}\left(\mathrm{H}_{2} \mathrm{O}\right)_{12}\right]^{7+}+\mathrm{H}_{2} \mathrm{AsO}_{4}^{-} \rightarrow$} & $\begin{array}{l}-413.94 \\
-409.88\end{array}$ & $\begin{array}{l}-24.7(\mathrm{M}-\mathrm{M}) \\
\text { (non } \\
\text { protonated) }\end{array}$ & $\begin{array}{l}\text { Fe-As } \\
3.22 \\
\text { Fe-Fe } \\
3.09 \\
\text { As-O } \\
1.61,1.75 \\
\text { As-OH } \\
1.78\end{array}$ \\
\hline \multirow[t]{3}{*}{$\begin{array}{l}\delta-\mathrm{Fe}_{13} \mathrm{Keggin} \\
\mathrm{As}^{5+}\left(\mathrm{HAsO}_{4}{ }^{2-}\right) \\
\text { binding }\end{array}$} & {$\left[\mathrm{Fe}_{13} \mathrm{O}_{4}(\mathrm{OH})_{24}\left(\mathrm{H}_{2} \mathrm{O}\right)_{12}\right]^{7+}+\mathrm{HAsO}_{4}^{2-} \rightarrow$} & $\begin{array}{l}-858.55 \\
-863.98\end{array}$ & $\begin{array}{l}-57.8(\mathrm{~B}-\mathrm{B}) \\
\text { (non } \\
\text { protonated) }\end{array}$ & $\begin{array}{l}\text { Fe-As } \\
3.20 \\
\text { Fe-Fe } \\
3.06 \\
\text { As-O } \\
1.61,1.76 \\
\text { As-OH } \\
1.79\end{array}$ \\
\hline & {$\left[\mathrm{Fe}_{13} \mathrm{O}_{4}(\mathrm{OH})_{24}\left(\mathrm{H}_{2} \mathrm{O}\right)_{10} \mathrm{AsO}_{3}(\mathrm{OH})\right]^{5+}+2 \mathrm{H}_{2} \mathrm{O}$} & & & \\
\hline & {$\left[\mathrm{Fe}_{13} \mathrm{O}_{4}(\mathrm{OH})_{24}\left(\mathrm{H}_{2} \mathrm{O}\right)_{12}\right]^{7+}+\mathrm{HAsO}_{4}^{2-} \rightarrow$} & $\begin{array}{l}-445.95 \\
-450.49\end{array}$ & $\begin{array}{l}7.6(\mathrm{M}-\mathrm{B}) \\
\text { (non } \\
\text { protonated) }\end{array}$ & $\begin{array}{l}\text { Fe-As } \\
2.81 \\
\text { Fe-Fe } \\
3.13 \\
\text { As-O } \\
1.66-1.78 \\
\text { As-OH } \\
1.72\end{array}$ \\
\hline
\end{tabular}




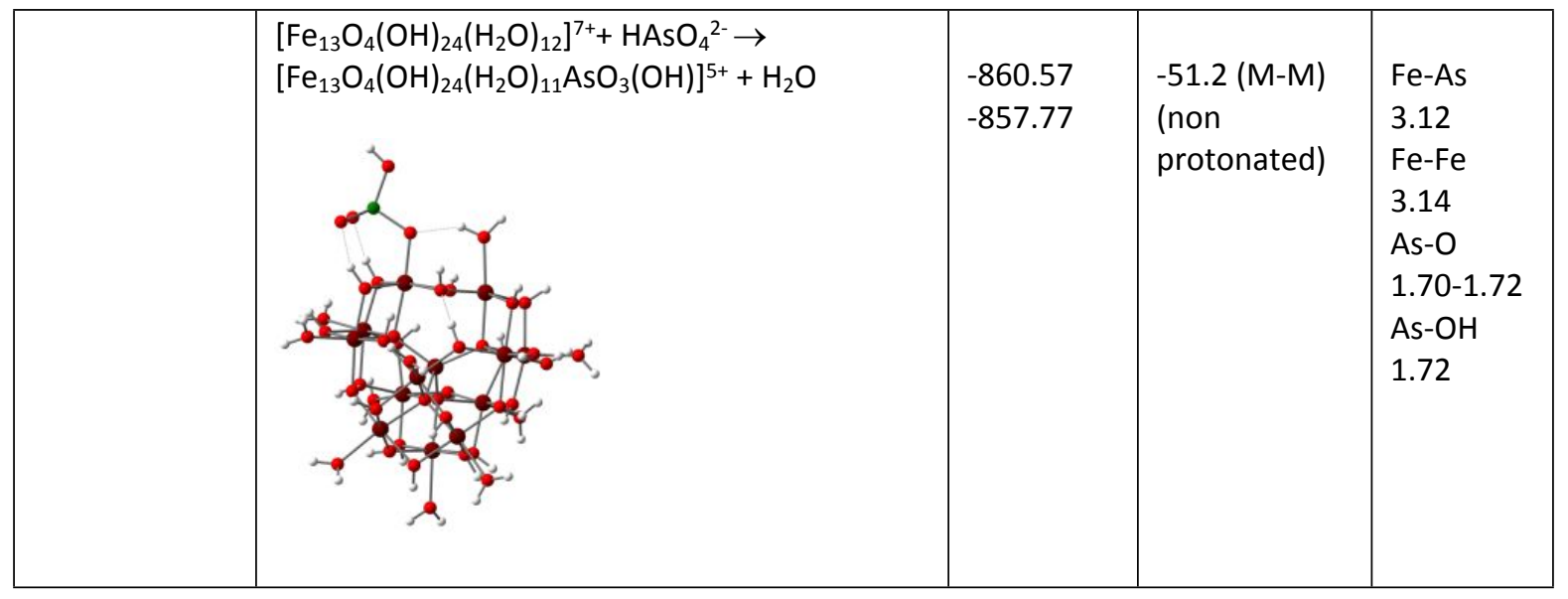

Scheme-S3.The optimized structures for reaction product of keggin cluster with $\mathrm{As}(\mathrm{OH})_{3}$ resulting inM-M complex $\left[\mathrm{Fe}_{13} \mathrm{O}_{4}(\mathrm{OH})_{24}\left(\mathrm{H}_{2} \mathrm{O}\right)_{11} \mathrm{As}(\mathrm{OH})_{3}\right]^{7+}$, which is converted to $\mathrm{B}-\mathrm{B}$ and $\mathrm{M}-\mathrm{B}$ products. Below the reactions are tabulated with calculated Gibb's free energies in kcal.In these structures brown balls are Fe(III), red balls are oxygen, and small greyish balls are hydrogen.

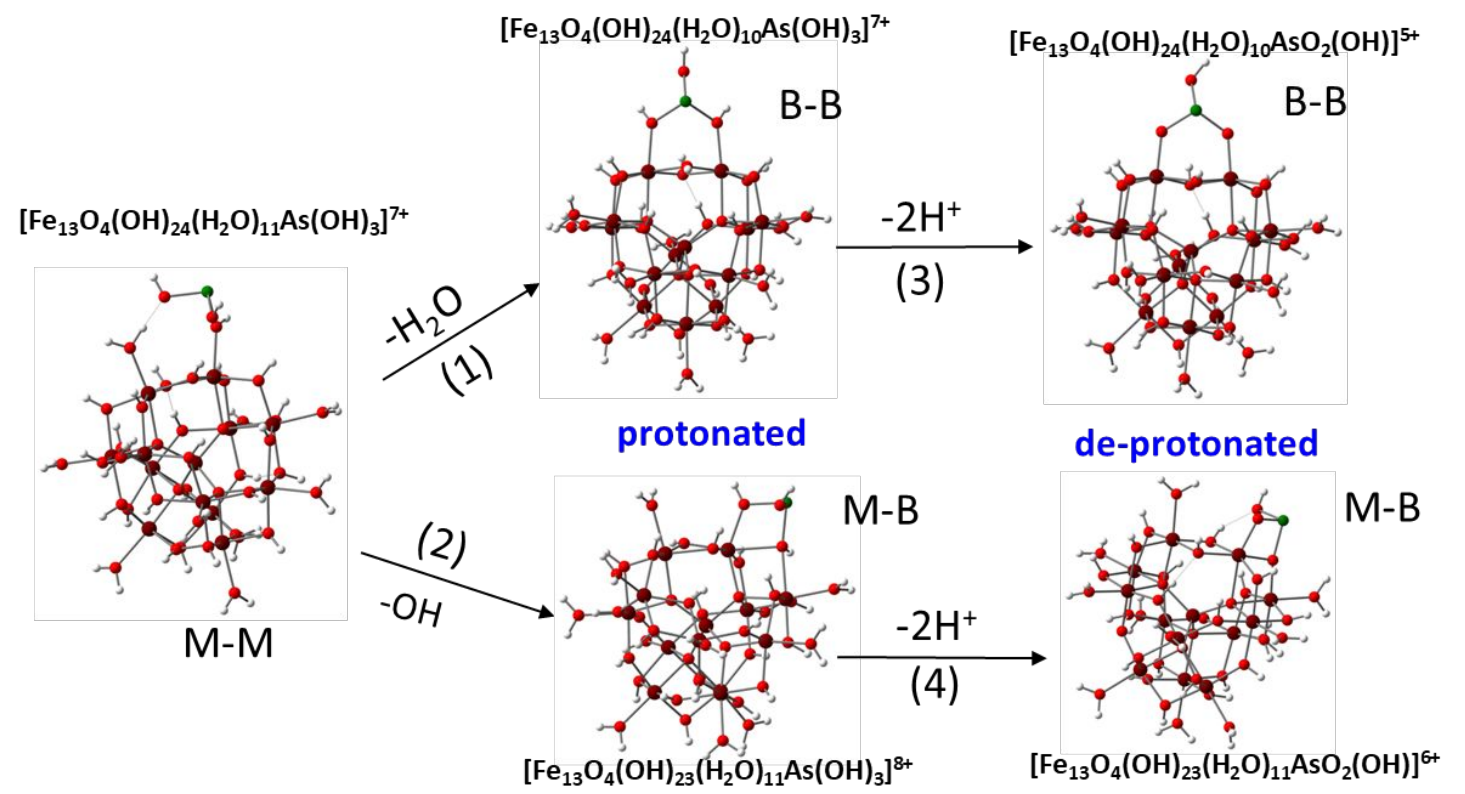

\begin{tabular}{|c|c|c|c|}
\hline Reactions & $\begin{array}{c}\Delta \mathrm{H} \text { (gas) } \\
\text { kcal }\end{array}$ & $\begin{array}{c}\Delta G \text { (gas) } \\
\text { kcal }\end{array}$ & $\begin{array}{l}\Delta \mathrm{Gs} \\
\mathrm{kca}\end{array}$ \\
\hline (1) $\left[\mathrm{Fe}_{13} \mathrm{O}_{4}(\mathrm{OH})_{24}\left(\mathrm{H}_{2} \mathrm{O}\right)_{11} \mathrm{As}(\mathrm{OH})_{3}\right]^{7+}(\mathrm{M}-\mathrm{M}) \rightarrow\left[\mathrm{Fe}_{13} \mathrm{O}_{4}(\mathrm{OH})_{24}\left(\mathrm{H}_{2} \mathrm{O}\right)_{10} \mathrm{As}(\mathrm{OH})_{3}\right]^{7+}(\mathrm{B}-\mathrm{B})+\mathrm{H}_{2} \mathrm{O}$ & 33.3 & 23.6 & 2.5 \\
\hline$(2)\left[\mathrm{Fe}_{13} \mathrm{O}_{4}(\mathrm{OH})_{24}\left(\mathrm{H}_{2} \mathrm{O}\right)_{11} \mathrm{As}(\mathrm{OH})_{3}\right]^{7+}(\mathrm{M}-\mathrm{M}) \rightarrow\left[\mathrm{Fe}_{13} \mathrm{O}_{4}(\mathrm{OH})_{23}\left(\mathrm{H}_{2} \mathrm{O}\right)_{11} \mathrm{As}(\mathrm{OH})_{3}\right]^{8+}(\mathrm{M}-\mathrm{B})+\mathrm{OH}^{-}$ & 587.1 & 575.7 & 72.9 \\
\hline (3) $\left[\mathrm{Fe}_{13} \mathrm{O}_{4}(\mathrm{OH})_{24}\left(\mathrm{H}_{2} \mathrm{O}\right)_{10} \mathrm{As}(\mathrm{OH})_{3}\right]^{7+}+2 \mathrm{H}_{2} \mathrm{O} \rightarrow\left[\mathrm{Fe}_{13} \mathrm{O}_{4}(\mathrm{OH})_{24}\left(\mathrm{H}_{2} \mathrm{O}\right)_{10} \mathrm{AsO}_{2}(\mathrm{OH})\right]^{5+}+2 \mathrm{H}_{3} \mathrm{O}+$ & -372.8 & -376.3 & 31.5 \\
\hline (4) $\left[\mathrm{Fe}_{13} \mathrm{O}_{4}(\mathrm{OH})_{23}\left(\mathrm{H}_{2} \mathrm{O}\right)_{11} \mathrm{As}(\mathrm{OH})_{3}\right]^{8+}+2 \mathrm{H}_{2} \mathrm{O} \rightarrow\left[\mathrm{Fe}_{13} \mathrm{O}_{4}(\mathrm{OH})_{23}\left(\mathrm{H}_{2} \mathrm{O}\right)_{11} \mathrm{AsO}_{2}(\mathrm{OH})\right]^{6+}+2 \mathrm{H}_{3} \mathrm{O}^{+}$ & -520.5 & -520.5 & $20 . C$ \\
\hline
\end{tabular}




\section{Gibb's free energies for $\mathrm{HAsO}_{4}{ }_{4}{ }^{2-i}$ ion binding to $\mathrm{Fe}$ (III) oxyhydroxide clusters}

The formation free energies of binding of Al doped and undoped pure Fe(III) oxyhydroxide clusters in presence of $\mathbf{H A s O}_{4}{ }^{2-}$ ion are listed below.

Table S20. The reactions of $\mathrm{Fe}(\mathrm{III})$ and $\mathrm{Al}(\mathrm{III})$ oxyhydroxide clusters with $\mathrm{HAsO}_{4}{ }^{2-}$ in gas phase and in water are shown. For each complexation cases, both the bidentate-binuclear and bidentate mononuclear type have been tabulated. In all cases, $\mathrm{Fe}^{3+}$ complexes are high-spin. The reaction free energies in aqueous medium are calculated form solvation free energies and corrected for standard states. ${ }^{17}$

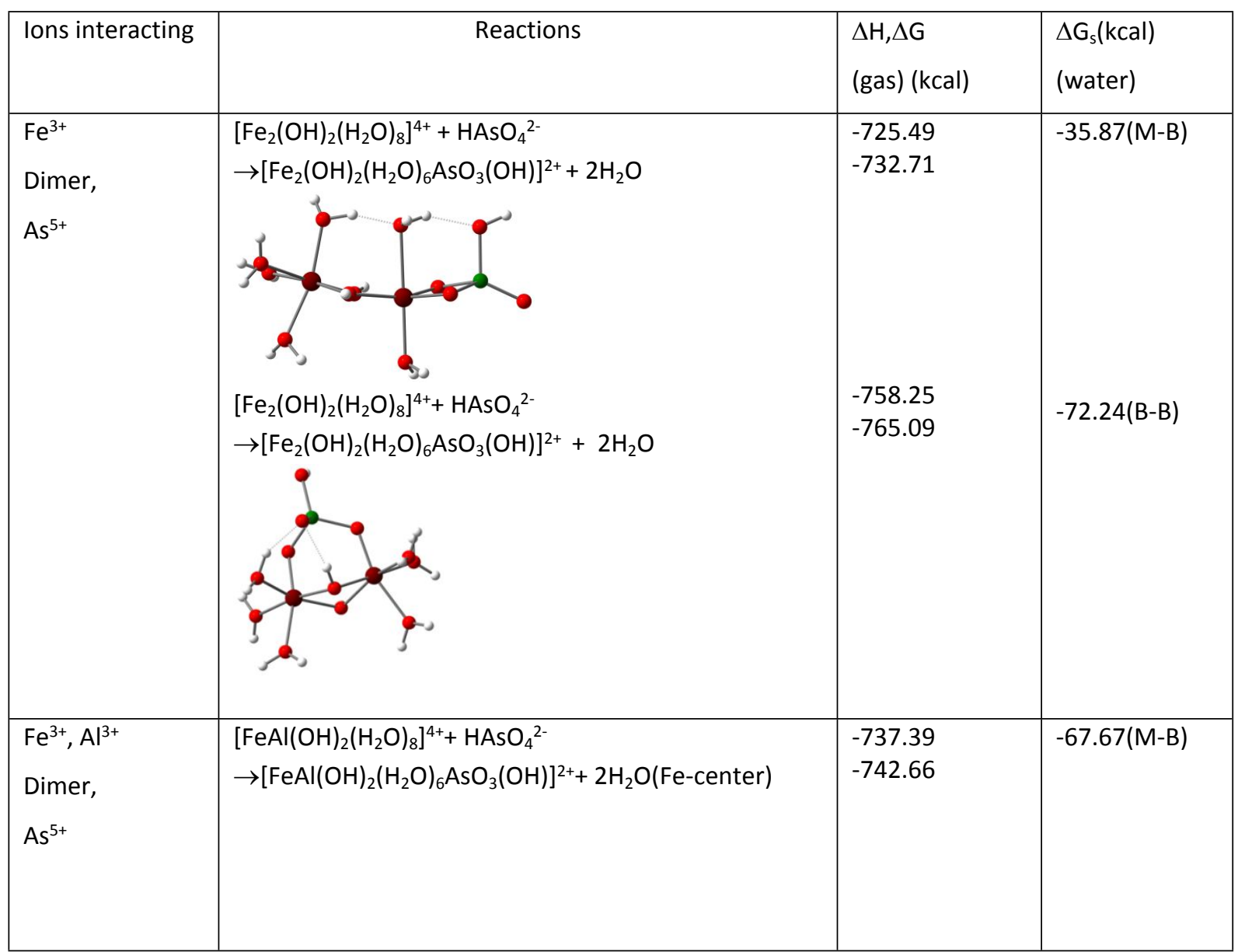




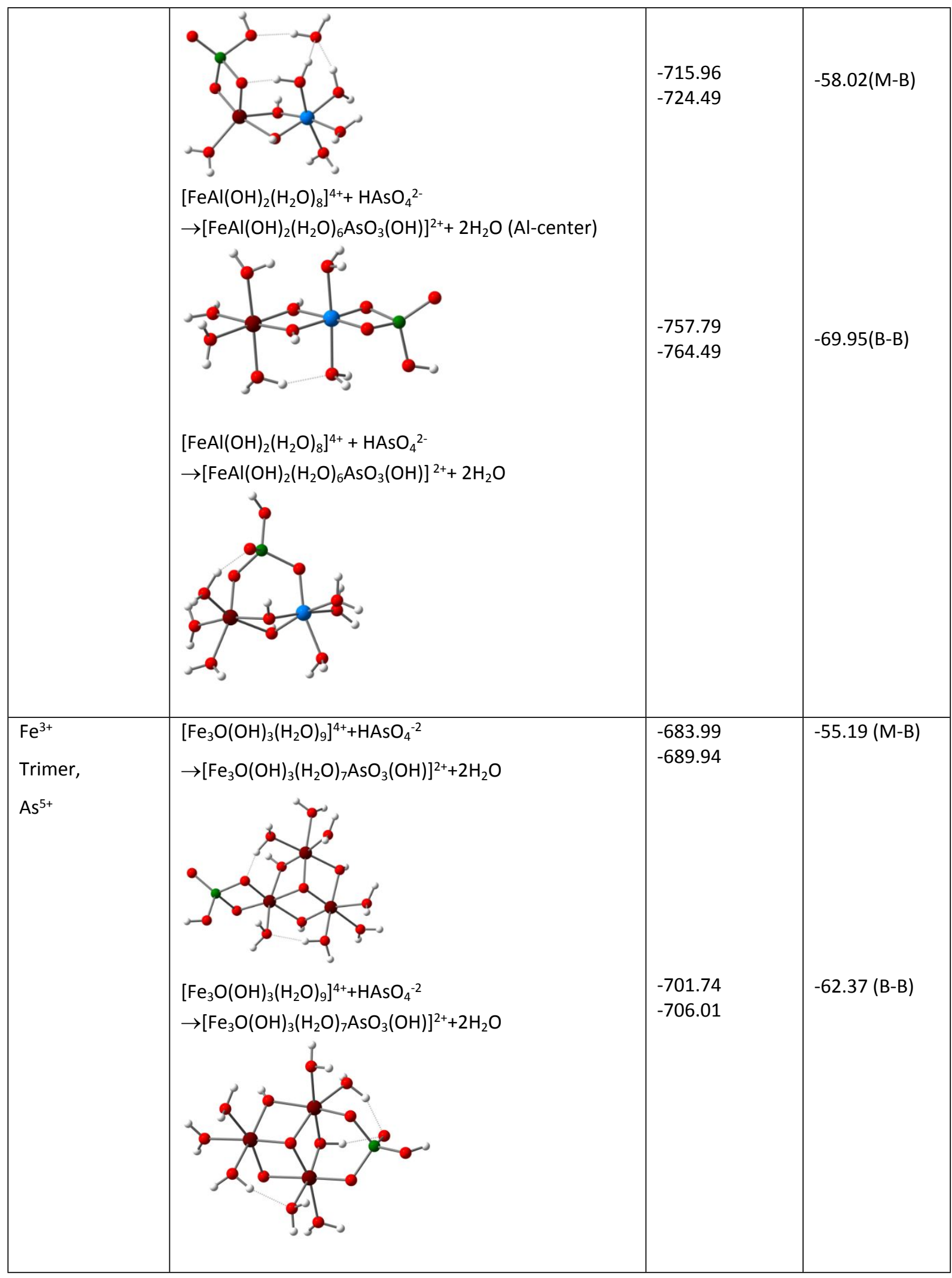




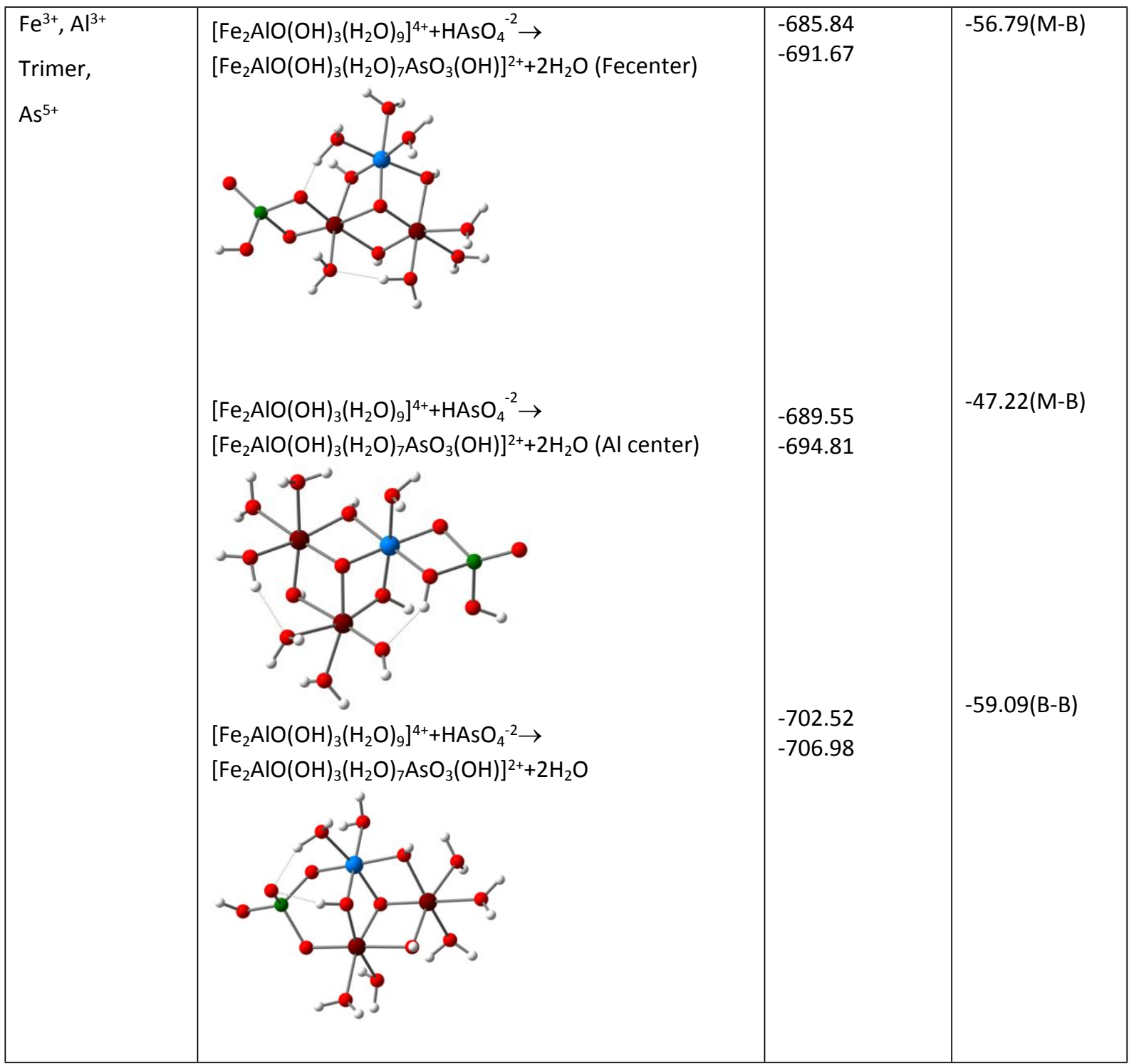

\section{Reactions of Keggin ion (doped/undoped) with $\mathrm{HAsO}_{4}{ }^{2-}$}

Table S21. The reactions of $\mathrm{Fe}(\mathrm{III})$ and $\mathrm{Al}(\mathrm{III})$ doped $\delta$ - $\mathrm{Fe}_{13} \mathrm{Keggin}$ clusters with $\mathrm{HAsO}_{4}{ }^{2-}$ in gas phase and in water are shown. The $\mathrm{Fe}^{3+} \delta-\mathrm{Fe}_{13} \mathrm{Keggin}$ clusters are high-spin. The reaction free energies in aqueous medium are calculated form solvation free energies and corrected for standard states. ${ }^{17}$

\begin{tabular}{|c|c|c|c|c|}
\hline Ions interacting & Reactions & $\begin{array}{l}\Delta H \text { (gas) } \\
\quad \text { (kcal) }\end{array}$ & $\begin{array}{c}\Delta G \text { (gas) } \\
\text { (kcal) }\end{array}$ & $\begin{array}{l}\Delta \mathrm{G}_{\mathrm{s}}(\mathrm{kcal}) \\
\text { (water) }\end{array}$ \\
\hline $\begin{array}{l}\delta-\mathrm{Fe}_{13} \mathrm{Keggin} \\
\mathrm{As}^{5+}\left(\mathrm{HAsO}_{4}{ }^{2-}\right) \\
\text { binding }\end{array}$ & $\begin{array}{l}\mathrm{Fe}_{13} \mathrm{O}_{4}(\mathrm{OH})_{24}\left(\mathrm{H}_{2} \mathrm{O}\right)_{12}{ }^{7+}+\mathrm{HAsO}_{4}{ }^{2-} \rightarrow \\
\mathrm{Fe}_{13} \mathrm{O}_{4}(\mathrm{OH})_{23}\left(\mathrm{H}_{2} \mathrm{O}\right)_{11} \mathrm{AsO}_{3}(\mathrm{OH})^{6+}+\mathrm{H}_{2} \mathrm{O} \\
+\mathrm{OH}^{-} \\
\mathrm{Fe}_{13} \mathrm{O}_{4}(\mathrm{OH})_{24}\left(\mathrm{H}_{2} \mathrm{O}\right)_{12}{ }^{7+}+\mathrm{HAsO}_{4}{ }^{2-} \rightarrow \\
\mathrm{Fe}_{13} \mathrm{O}_{4}(\mathrm{OH})_{24}\left(\mathrm{H}_{2} \mathrm{O}\right)_{10} \mathrm{AsO}_{3}(\mathrm{OH})^{5+}+2 \mathrm{H}_{2} \mathrm{O}\end{array}$ & $\begin{array}{l}-445.95 \\
-858.55\end{array}$ & $\begin{array}{l}-450.49 \\
-863.98\end{array}$ & $\begin{array}{l}7.56(\mathrm{M}-\mathrm{B}) \\
-57.84(\mathrm{~B}-\mathrm{B})\end{array}$ \\
\hline $\begin{array}{l}\text { Case I } \\
\delta \text {-Fe }{ }_{12} \text { AlKeggin } \\
\end{array}$ & $\begin{array}{l}\mathrm{Fe}_{12} \mathrm{AlO}_{4}(\mathrm{OH})_{24}\left(\mathrm{H}_{2} \mathrm{O}\right)_{12}{ }^{7+}+\mathrm{HAsO}_{4}{ }^{2-} \rightarrow \\
\mathrm{Fe}_{12} \mathrm{AlO}_{4}(\mathrm{OH})_{23}\left(\mathrm{H}_{2} \mathrm{O}\right)_{11} \mathrm{AsO}_{3}(\mathrm{OH})^{6+}+\end{array}$ & -445.29 & -451.17 & 7.69 (M-B) \\
\hline
\end{tabular}




\begin{tabular}{|c|c|c|c|c|}
\hline $\begin{array}{l}\text { cluster (doped by } \\
\text { single } \mathrm{Al}^{3+} \text { on } \mathrm{Fe}^{1} \\
\text { site), } \mathrm{As}^{5+} \text { bound to } \\
\text { Fe-Al site }\end{array}$ & $\begin{array}{l}\mathrm{H}_{2} \mathrm{O}+\mathrm{OH}^{-} \\
\\
\mathrm{Fe}_{12} \mathrm{AlO}_{4}(\mathrm{OH})_{24}\left(\mathrm{H}_{2} \mathrm{O}\right)_{12}{ }^{7+}+\mathrm{HAsO}_{4}{ }^{2-} \rightarrow \\
\mathrm{Fe}_{12} \mathrm{AlO}_{4}(\mathrm{OH})_{24}\left(\mathrm{H}_{2} \mathrm{O}\right)_{10} \mathrm{AsO}_{3}(\mathrm{OH})^{5+}+ \\
2 \mathrm{H}_{2} \mathrm{O}\end{array}$ & -855.54 & -862.44 & -54.35 (B-B) \\
\hline $\begin{array}{l}\text { Case II } \\
\delta \text {-Fe }{ }_{12} \mathrm{AlKeggin} \\
\text { cluster(doped by } \\
\text { single } \mathrm{Al}^{3+} \text { on } \\
\mathrm{Fe}^{1} \mathrm{site}^{3}, \mathrm{As}^{5+} \text { bound } \\
\text { on Fe-Fe site far } \\
\text { away from doped } \\
\text { site) }\end{array}$ & $\begin{array}{l}\mathrm{Fe}_{12} \mathrm{AlO}_{4}(\mathrm{OH})_{24}\left(\mathrm{H}_{2} \mathrm{O}\right)_{12}{ }^{7+}+\mathrm{HAsO}_{4}{ }^{2-} \rightarrow \\
\mathrm{Fe}_{12} \mathrm{AlO}_{4}(\mathrm{OH})_{23}\left(\mathrm{H}_{2} \mathrm{O}\right)_{11} \mathrm{AsO}_{3}(\mathrm{OH})^{6+}+ \\
\mathrm{H}_{2} \mathrm{O}+\mathrm{OH}^{-} \\
\mathrm{Fe}_{12} \mathrm{AlO}_{4}(\mathrm{OH})_{24}\left(\mathrm{H}_{2} \mathrm{O}\right)_{12}{ }^{7+}+\mathrm{HAsO}_{4}{ }^{2-} \\
\rightarrow \mathrm{Fe}_{12} \mathrm{AlO}_{4}(\mathrm{OH})_{24}\left(\mathrm{H}_{2} \mathrm{O}\right)_{10} \mathrm{AsO}_{3}(\mathrm{OH})^{5+}+ \\
2 \mathrm{H}_{2} \mathrm{O}\end{array}$ & -860.03 & -867.20 & 7.89(M-B) \\
\hline $\begin{array}{l}\text { Case III } \\
\delta \text {-Fe }{ }_{12} \mathrm{Al} \text { Keggin } \\
\text { cluster (doped by } \\
\text { single } \mathrm{Al}^{3+} \text { on } \mathrm{Fe}^{3} \\
\text { site), } \mathrm{As}^{5+} \text { bound to } \\
\text { Fe-Fe site }\end{array}$ & $\begin{array}{l}\mathrm{Fe}_{12} \mathrm{AlO}_{4}(\mathrm{OH})_{24}\left(\mathrm{H}_{2} \mathrm{O}\right)_{12}{ }^{7+}+\mathrm{HAsO}_{4}{ }^{2-} \rightarrow \\
\mathrm{Fe}_{12} \mathrm{AlO}_{4}(\mathrm{OH})_{23}\left(\mathrm{H}_{2} \mathrm{O}\right)_{11} \mathrm{AsO}_{3}(\mathrm{OH})^{6+}+ \\
\mathrm{H}_{2} \mathrm{O}+\mathrm{OH}^{-} \\
\mathrm{Fe}_{12} \mathrm{AlO}_{4}(\mathrm{OH})_{24}\left(\mathrm{H}_{2} \mathrm{O}\right)_{12}{ }^{7+}+\mathrm{HAsO}_{4}{ }^{2-} \\
\rightarrow \mathrm{Fe}_{12} \mathrm{AlO}_{4}(\mathrm{OH})_{24}\left(\mathrm{H}_{2} \mathrm{O}\right)_{10} \mathrm{AsO}_{3}(\mathrm{OH})^{5+}+ \\
2 \mathrm{H}_{2} \mathrm{O}\end{array}$ & $\begin{array}{l}-439.86 \\
-861.64\end{array}$ & $\begin{array}{l}-444.13 \\
-867.53\end{array}$ & -55.83 (B-B) \\
\hline $\begin{array}{l}\text { Case IV } \\
\delta \text {-Fe }{ }_{12} \mathrm{Al} \text { Keggin } \\
\text { cluster (doped by } \\
\text { two } \mathrm{Al}^{3+} \text { on two } \mathrm{Fe}^{1} \\
\text { sites), } \mathrm{As}^{5+} \text { bound } \\
\text { to Al-Al site }\end{array}$ & $\begin{array}{l}\mathrm{Fe}_{11} \mathrm{Al}_{2} \mathrm{O}_{4}(\mathrm{OH})_{24}\left(\mathrm{H}_{2} \mathrm{O}\right)_{12}{ }^{7+}+\mathrm{HAsO}_{4}{ }^{2-} \rightarrow \\
\mathrm{Fe}_{11} \mathrm{Al}_{2} \mathrm{O}_{4}(\mathrm{OH})_{23}\left(\mathrm{H}_{2} \mathrm{O}\right)_{11} \mathrm{AsO}_{3}(\mathrm{OH})^{6+}+ \\
\mathrm{H}_{2} \mathrm{O}+\mathrm{OH}^{-} \\
\mathrm{Fe}_{11} \mathrm{Al}_{2} \mathrm{O}_{4}(\mathrm{OH})_{24}\left(\mathrm{H}_{2} \mathrm{O}\right)_{12}{ }^{7+}+\mathrm{HAsO}_{4}{ }^{2-} \rightarrow \\
\mathrm{Fe}_{11} \mathrm{Al}_{2} \mathrm{O}_{4}(\mathrm{OH})_{24}\left(\mathrm{H}_{2} \mathrm{O}\right)_{10} \mathrm{AsO}_{3}(\mathrm{OH})^{5+}+ \\
2 \mathrm{H}_{2} \mathrm{O}\end{array}$ & -850.81 & -857.41 & $-51.28(B-B)$ \\
\hline
\end{tabular}

